\title{
Hydrogen molecules in a superstrong magnetic field: Excitation levels
}

\author{
Dong Lai* \\ Theoretical Astrophysics, 130-33, California Institute of Technology, Pasadena, California 91125 \\ Edwin E. Salpeter \\ Center for Radiophysics and Space Research, Cornell University, Ithaca, New York 14853
}

(Received 3 August 1995)

\begin{abstract}
We study the energy levels of $\mathrm{H}_{2}$ molecules in a superstrong magnetic field ( $B \gtrsim 10^{12} \mathrm{G}$ ), typically found on the surfaces of neutron stars. The interatomic interaction potentials are calculated by a Hartree-Fock method with multiconfigurations assuming electrons are in the ground Landau state. Both the aligned configurations and arbitrary orientations of the molecular axis with respect to the magnetic-field axis are considered. Different types of molecular excitations are then studied: electronic excitations, aligned (along the magnetic axis) vibrational excitations, and transverse vibrational excitations (a constrained rotation of the molecular axis around the magnetic-field line). Similar results for the molecular ion $\mathrm{H}_{2}^{+}$are also obtained and compared with previous variational calculations. Both numerical results and analytical fitting formulas are given for a wide range of field strengths. In contrast to the zero-field case, it is found that the transverse vibrational excitation energies can be larger than the aligned vibration excitation, and they both can be comparable to or larger than the electronic excitations. For $B \geq B_{c r i t}=4.23 \times 10^{13} \mathrm{G}$, the Landau energy of the proton is appreciable and there is some controversy regarding the dissociation energy of $\mathrm{H}_{2}$. We show that $\mathrm{H}_{2}$ is bound even for $B$ $\gg B_{\text {crit }}$ and that neither proton has a Landau excitation in the ground molecular state.
\end{abstract}

PACS number(s): 32.60.+i, 97.10.Ld, 31.15.Ar, 97.60.Jd

\section{INTRODUCTION}

Since the pioneering work of Schiff and Snyder [1], especially during the last 20 years, there has been considerable interest in the properties of matter in a strong magnetic field. While the early studies [2] were mainly motivated by the fact that high magnetic-field conditions can be mimicked in some semiconductors where a small effective mass and a large dielectric constant reduce the electric force relative to the magnetic force, the recent interest in this problem has been motivated by the huge magnetic field $\sim 10^{12} \mathrm{G}$ already discovered in many neutron stars and the tentative suggestion for fields as strong as $10^{15} \mathrm{G}$. The surface layer of these neutron stars then consists of highly magnetized matter. Understanding the physical properties of atoms, molecular chains, and condensed matter in fields of such extreme magnitude (see Ref. [3] for an early general review and [4] for a recent text on atoms in strong magnetic fields) is important for interpreting the radiation from the neutron stars that may be observed in the present and future x-ray satellites (e.g., [5]), and therefore provides important information about the internal structure of neutron stars.

In superstrong magnetic fields the structure of atoms and condensed matter is dramatically changed by the fact that the magnetic force on an electron is stronger than the Coulomb force it experiences, i.e., the electron cyclotron energy (the Landau energy level spacing)

$$
\hbar \omega_{e}=\hbar \frac{e B}{m_{e} c}=11.57 B_{12} \mathrm{keV}
$$

\footnotetext{
*Electronic address: dong@tapir.caltech.edu
}

where $B_{12}$ is the magnetic-field strength in units of $10^{12} \mathrm{G}$, is much larger than the typical Coulomb energy. In the direction perpendicular to the field, the electrons are confined to move on cylindrical Landau orbitals around a nucleus. The orbitals have radii

$$
\rho_{m}=(2 m+1)^{1 / 2} \hat{\rho}, \quad m=0,1,2, \ldots,
$$

where $\hat{\rho}$ is the cyclotron radius

$\hat{\rho}=\left(\frac{\hbar c}{e B}\right)^{1 / 2}=a_{0}\left(\frac{B_{0}}{B}\right)^{1 / 2}=2.57 \times 10^{-10} B_{12}^{-1 / 2} \mathrm{~cm}$.

Here $a_{0}=\hbar^{2} /\left(m_{e} c^{2}\right)$ is the Bohr radius and $B_{0}$ is the atomic unit for the magnetic-field strength,

$$
B_{0}=\frac{m_{e}^{2} e^{3} c}{\hbar^{3}}=2.35 \times 10^{9} \mathrm{G}, \quad b \equiv \frac{B}{B_{0}}=425 B_{12} .
$$

Throughout this paper we consider strong fields in the sense of $b \gg 1$, so that the Coulomb forces act as a perturbation to the magnetic forces on the electrons, and the electrons are confined to the ground Landau level (so-called "adiabatic approximation" [1]). Because of this extreme confinement of electrons in the transverse direction, the Coulomb force becomes much more effective for binding electrons in the parallel direction, therefore giving greatly increased binding energy. The atom has a cigarlike structure. Moreover, it is possible for these elongated atoms to form molecular chains by covalent bonding along the field direction $[3,6]$.

Significant efforts have been devoted to the theoretical study of atoms in a superstrong magnetic field $\left(\gtrsim 10^{12} \mathrm{G}\right)$ [4]. The methods that have been employed include variational calculations (e.g., [7]), Thomas-Fermi-type statistical 
models [8], density functional theory [9], and the selfconsistent Hartree-Fock method [10-12], which we consider to be the more theoretically justified and reliable method. Accurate calculations of the energy levels of the $\mathrm{H}$ atom in magnetic fields of arbitrary strength have also been performed [13]. By contrast, there are only limited studies on molecules in superstrong magnetic field; nearly all of these focus on the molecular ion $\mathrm{H}_{2}^{+}$( [14-17] and references therein). $\mathrm{As}_{2}{ }^{+}$is unstable against forming $\mathrm{H}_{2}$, understanding the physical properties of a $\mathrm{H}_{2}$ molecule is of greater practical interest, since $\mathrm{H}_{2}$ is likely to exist in the atmosphere of sufficiently cool neutron stars $[18,19]$.

We have recently calculated the ground-state binding energies of different forms of hydrogen $\left(\mathrm{H}, \mathrm{H}^{-}, \mathrm{H}_{2}{ }^{+}, \mathrm{H}_{2}\right.$, $\left.\mathrm{H}_{3}, \ldots, \mathrm{H}_{\infty}\right)$ in a strong magnetic field $B \geq 10^{12} \mathrm{G}([6]$, hereafter referred to as paper I). In particular, reliable electronic dissociation energy of a $\mathrm{H}_{2}$ molecule in magnetic field of such magnitude was obtained. In this paper, we extend our study to consider various excitation levels of the molecule.

In the zero-field case, to study the molecular spectra, one usually uses the Born-Oppenheimer approximation to separate the motion of the ions from that of the electrons. Such a procedure is valid if the electronic energy-level spacings are large compared to the typical energy-level spacings associated with the ion motion. In a strong magnetic field, however, the separation of motion becomes much more complicated, even for the hydrogen atom [20-22]. Moreover, as we shall see, in a superstrong magnetic field, the energy-level spacings associated with the vibrations of the ions can be comparable to or even larger than the spacings of the electronic excitations. In this paper, we will use the standard Born-Oppenheimer approximation and focus on calculating the interatomic interaction potential for fixed ion positions (Sec. III). We then obtain the molecular excitation levels based on this potential curve (Sec. IV). As in the case of a neutral atom [20], it is convenient to define a critical field strength by equating the cyclotron energy of the proton $\hbar \omega_{p}=\hbar\left(e B / m_{p} c\right)$ to the typical electronic excitation energy ( $\sim \ln b$ in atomic units), i.e.,

$$
\begin{aligned}
b_{\text {crit }} & \equiv \frac{m_{p}}{m_{e}} \ln b_{\text {crit }}=1.80 \times 10^{4} ; \\
B_{\text {crit }} & =b_{\text {crit }} B_{0}=4.23 \times 10^{13} \mathrm{G} .
\end{aligned}
$$

We shall give quantitative results for the regime $B_{0} \ll B$ $\ll B_{c r i t}$ in Secs. II-IV, using the standard Born-Oppenheimer procedure. Rigorous calculations for the molecule when $B \gtrsim B_{\text {crit }}$, taking account of the quantum mechanics of the proton motion, are difficult. Nevertheless, in Sec. V we shall describe an approximate solution to the four-body problem of the $\mathrm{H}_{2}$ molecule in the $B \gg B_{\text {crit }}$ regime, where the effects of finite proton mass on the electronic states and the energies of the molecule are strong, and we give a rigorous lower limit to the ground-state dissociation energy.

Throughout this paper, we shall use nonrelativistic quantum mechanics, even for extremely strong magnetic field, $B \geq B_{r e l}=\left(\hbar c / e^{2}\right)^{2} B_{0}=4.414 \times 10^{13} \mathrm{G}$ (note that $B_{r e l}$ is close to $B_{\text {crit }}$ only by coincidence), at which the transverse motion of the electron becomes relativistic. The nonrelativ- istic treatment of bound states is valid for two reasons: (i) the energy of a relativistic free electron

$$
E=\left[c^{2} p_{z}^{2}+m_{e}^{2} c^{4}\left(1+2 n_{L} B / B_{r e l}\right)\right]^{1 / 2},
$$

where $p_{z}$ is the linear momentum along the field axis, $n_{L}$ is the quantum number for the Landau excitations, reduces to $E \simeq m_{e} c^{2}+p_{z}^{2} /\left(2 m_{e}\right)$ as long as the electron remains in the ground Landau level and nonrelativistic in the $z$ direction; (ii) the shape of the Landau wave function in the relativistic theory is the same as in the nonrelativistic theory (as we see from the fact that $\hat{\rho}$ is independent of mass). Therefore, as long as $E_{B} /\left(m_{e} c^{2}\right) \ll 1$, where $E_{B}$ is the binding energy of the bound state, the relativistic effect remains a small correction [23].

The paper is organized as follows. In Sec. II we consider some general features and approximate scaling relations for various excitation levels. Section III contains a detailed description of our method for calculating the interatomic interaction potential. The numerical results and fitting formulas for the molecular excitation levels are presented in Sec. IV. We study the electronic structure of the molecule in the $B$ $\gg B_{\text {crit }}$ regime and consider the effects of finite proton mass on the energies in Sec. V. Our general conclusion is presented in Sec. VI. Appendix A summarizes some useful mathematical relations for the Coulomb integrals of Landau functions, and in Appendix B we discuss a refined method for calculating the electronic energy of $\mathrm{H}_{2}{ }^{+}$for general orientation of the molecular axis.

\section{QUALITATIVE DISCUSSION AND APPROXIMATE SCALING RELATIONS FOR EXCITATION ENERGIES}

In a superstrong magnetic field satisfying $b \gg 1$, the spectra of a single $\mathrm{H}$ atom can be specified by two quantum numbers $(m, \nu)$, where $m$ measures the mean transverse distance [Eq. (1.2)] of the electron to the proton, while $\nu$ is the number of nodes of the electron's $z$ wave function (along the field direction). The wave function of the $(m, \nu)$ state in cylindrical coordinates $(\rho, \phi, z)$ is given by

$$
\Phi_{m \nu}=W_{m}\left(\mathbf{r}_{\perp}\right) f_{m \nu}(z),
$$

where $W_{m}$ is the ground-state Landau wave function

$$
W_{m}\left(\mathbf{r}_{\perp}\right) \equiv W_{m}(\rho, \phi)=\frac{1}{\hat{\rho} \sqrt{2 \pi m !}}\left(\frac{\rho}{\hat{\rho} \sqrt{2}}\right)^{m} e^{-\rho^{2} / 4 \hat{\rho}^{2}} e^{-i m \phi} .
$$

The states with $\nu \neq 0$ resemble a zero-field hydrogen atom with small binding energy $\left|E_{\nu}\right| \simeq 1 /\left(2 \nu^{2}\right)[24]$ and we shall mostly focus on the tightly bound states with $\nu=0$. For the ground state $(0,0)$, the sizes $L_{\perp}$ and $L_{z}$ of the atomic wave function perpendicular and parallel to the field and the binding energy $\left|E_{a}\right|$ (in atomic units) are given by

$$
L_{\perp} \sim \hat{\rho}=\frac{1}{b^{1 / 2}}, \quad L_{z} \sim \frac{1}{l}, \quad\left|E_{a}\right| \simeq 0.16 l^{2} ; \quad l \equiv \ln b .
$$


TABLE I. Energy levels $E_{a}(m)$ (in eV) of a hydrogen atom in a superstrong magnetic field. The levels are specified by the quantum number $m$, while the longitudinal node $\nu=0$. Here $B_{12}=B /\left(10^{12} \mathrm{G}\right)$.

\begin{tabular}{ccccccc}
\hline \hline$B_{12}$ & $E_{a}(0)$ & $E_{a}(1)$ & $E_{a}(2)$ & $E_{a}(3)$ & $E_{a}(4)$ & $E_{a}(5)$ \\
\hline 0.1 & -76.4 & -52.5 & -43.3 & -38.0 & -34.4 & -31.8 \\
0.5 & -130.2 & -92.8 & -77.8 & -69.0 & -63.0 & -58.6 \\
1 & -161.5 & -116.9 & -98.7 & -88.0 & -80.6 & -75.1 \\
2 & -198.5 & -145.8 & -124.1 & -111.2 & -102.2 & -95.5 \\
5 & -257.1 & -192.6 & -165.5 & -149.2 & -137.8 & -129.2 \\
10 & -309.6 & -235.1 & -203.5 & -184.3 & -170.9 & -160.7 \\
\hline \hline
\end{tabular}

For the tightly bound excited states $(m, 0)$ we have similar relations but with $\hat{\rho}$ replaced by $[(2 m+1) / b]^{1 / 2}$ and $l$ replaced by $l_{m} \equiv \ln [b /(2 m+1)]$, so that

$$
E_{a}(m) \simeq-0.16 l_{m}^{2}
$$

Recall that in atomic units, mass is in units of the electron mass $m_{e}$, energy is expressed in units of $e^{2} / a_{0}=2 \mathrm{Ry}$, length is in units of Bohr radius $a_{0}$, and the units for magnetic field is $B_{0}$ [Eq. (1.4)]. The numerical factor 0.16 in Eqs. (2.3) and (2.4) is an approximate value for $B_{12} \gtrsim 1$. For convenience, accurate numerical results for $E_{a}(m)$ are listed in Table I. ${ }^{1}$

In a superstrong magnetic field, the mechanism of forming molecules is quite different from the zero-field case (paper I, [3]). The spins of the electrons of the atoms in a strong magnetic field are all aligned antiparallel to the magnetic field, and therefore two atoms in their ground states do not easily bind together according to the exclusion principle. Thus two $\mathrm{H}$ atoms, both in the $m=0$ ground state, do not form a tightly bound molecule. Instead, one $\mathrm{H}$ atom has to be excited to the $m=1$ state. The two $\mathrm{H}$ atoms, one in the ground state $(m=0)$, another in the $m=1$ state, then form the ground state of a $\mathrm{H}_{2}$ molecule by covalent bonding. Since the "activation energy" for exciting an electron in the $\mathrm{H}$ atom from Landau orbital $m$ to $(m+1)$ is small [see Eq. (2.4)], the resulting molecule is stable. The interatomic separation $Z_{0}$ and the dissociation energy $D$ of the $\mathrm{H}_{2}$ molecule scale approximately as

$$
Z_{0}=\xi L_{z} \sim \frac{\xi}{l}, \quad D \sim \frac{l}{Z_{0}} \sim \frac{l^{2}}{\xi},
$$

where the dimensionless factor $\xi$ decreases very slowly with increasing $B$ [e.g., $\xi \simeq 2.0$ for $B_{12}=0.1$ and $\xi \simeq 0.75$ for $B_{12}=100$; see Table I of paper I and our Eq. (5.2)].

Another mechanism of forming a $\mathrm{H}_{2}$ molecule in a superstrong magnetic field is to let both electrons occupy the same $m=0$ Landau state, while one of them occupies the $\nu=0$ orbital and another the $\nu=1$ orbital. This costs no "activation energy." However, the resulting molecule tends to have small dissociation energy, of order a Rydberg. We shall refer

\footnotetext{
${ }^{1}$ A more accurate fitting formula for the ground state binding energy of a $\mathrm{H}$ atom is $\left|E_{a}\right|=0.16 A l^{2}$, with

$$
A= \begin{cases}1+1.36 \times 10^{-2}[\ln (1000 / b)]^{2.5} & \text { if } b<10^{3} \\ 1+1.07 \times 10^{-2}[\ln (b / 1000)]^{1.6} & \text { if } b \geqslant 10^{3} .\end{cases}
$$
}

to this electronic state of the molecule as the weakly bound state, and to the states formed by two electrons in the $\nu=0$ orbitals as the tightly bound states. As we will see below, as long as $l \gg 1$, the weakly bound state only constitutes an excited energy level of the molecule. ${ }^{2}$

We now consider various molecular excitations and derive approximate scaling relations for the excitation energies.

\section{A. Electronic excitations}

The electronic excitations of $\mathrm{H}_{2}{ }^{+}$are similar to those of the $\mathrm{H}$ atom, namely the electron can occupy different $m$ Landau orbitals. Thus $m=0$ is the ground state, $m=1,2, \ldots$ are the excited states (although they are not necessarily bound relative to the free atom in the ground state).

There are two types of electronic excitations in $\mathrm{H}_{2}$. (i) The electrons can occupy different orbitals other than the ground state $\left(m_{1}, m_{2}\right)=(0,1)$, giving rise to the tightly bound $(\nu=0)$ electronic excitations. For example, the first excited level is $(0,2)$, the second excited level is $(0,3)$, etc. The number of single $m$-excitation states $\left(m_{1}, m_{2}\right)=\left(0, m_{2}\right)$ which are bound relative to two isolated $\mathrm{H}$ atoms in the ground state is expected to increase as the magnetic field increases. Double $m$ excitations are also possible, but as we shall see, they are bound only when the magnetic-field strength is much higher than $10^{13} \mathrm{G}$. The energy spacing between the two adjacent electronic states $(0, m)$ and $(0, m+1)$ is

$$
\Delta E_{m} \sim l \ln \left(\frac{2 m+3}{2 m+1}\right) .
$$

Thus as $m$ increases, the energy spacing decreases. (ii) The molecule is formed by two electrons in the $(m, \nu)=(0,0)$ and $(0,1)$ orbitals. The dissociation energy of this weakly bound state is of order a Rydberg, and does not depend sensitively on the magnetic-field strength. Note that for relatively small magnetic field ( $B_{12} \gtrsim 0.2$ ), the weakly bound state actually has lower energy than the tightly bound states (see Sec. IV

\footnotetext{
${ }^{2}$ In several recent papers [25] on the molecular binding in strong magnetic field, Korolev and Liberman failed to identify the tightly bound states. Also, their variational calculation of the weakly bound state significantly underestimates the binding energy because it neglects the overlapping of the electron wave functions. As a result, their claim that hydrogenlike gas in a strong magnetic field can form Bose-Einstein condensate is incorrect (see also [26,27]).
} 
A), i.e., $b \gtrsim 10^{2}$ is required for the "strong field" regime to apply fully.

\section{B. Aligned vibrations}

In the Born-Oppenheimer approximation, the motion of the two protons is governed by the interatomic potential $U\left(Z, R_{\perp}\right)$, i.e., the electronic energy when the relative positions of the protons are kept at $Z$ along the field direction and $R_{\perp}$ perpendicular to it. We first consider the aligned vibrational excitations for oscillations of $Z$ about the equilibrium separation $Z_{0}$. For this purpose we need to estimate the excess potential $\delta U(\delta Z) \equiv U\left(Z_{0}+\delta Z, 0\right)-U\left(Z_{0}, 0\right)$.

Since $Z_{0}$ is the equilibrium position, the sum of the first order terms in $\delta Z$, coming from proton-proton, electronelectron, proton-electron Coulomb energies, and quantum mechanical electron kinetic energy, must cancel. Thus we have $\delta U \propto(\delta Z)^{2}$ for small $\delta Z$. Consider various contributions to the energy of the molecule: The proton-proton interaction is $1 / Z$ (in atomic units) without a logarithmic factor; but the dominant contribution is the proton-electron Coulomb energy $\sim l / Z$, where the logarithmic factor $l \gg 1$ comes from the Coulomb integral over the "cigar-shaped" electron distribution. Both $l$ and $Z^{-1}$ change as $Z_{0} \rightarrow Z_{0}+\delta Z$, but the largest change comes from the quadratic term $\delta\left(Z^{-1}\right) \sim(\delta Z)^{2} / Z_{0}^{3}$. Thus the excess potential is of order

$$
\delta U(\delta Z) \sim l \frac{(\delta Z)^{2}}{Z_{0}^{3}} \sim\left(\xi^{-3} l^{4}\right)(\delta Z)^{2} .
$$

In atomic (electron) units the reduced mass of the proton pair in $\mathrm{H}_{2}$ is $\mu=m_{p} /\left(2 m_{e}\right)$, where $m_{p}$ and $m_{e}$ are proton and electron mass (for HD the factor $1 / 2$ is replaced by 2/3). For small-amplitude oscillations in the potential of Eq. (2.7), we obtain a harmonic oscillation spectrum with excitation energy quanta $\hbar \omega_{\|}$given by

$$
\hbar \omega_{\|} \sim \xi^{-3 / 2} l^{2} \mu^{-1 / 2}
$$

for a molecule in the ground electronic state. The scaling with $B$ of $\hbar \omega_{\|}$is thus almost the same as the dissociation energy $D$ in Eq. (2.5). The number of aligned vibrational levels is $n_{\| \max } \sim D / \hbar \omega_{\|} \sim(\xi \mu)^{1 / 2}$, where $\xi$ decreases even more slowly with increasing field strength than $l^{-1}$ does.

\section{Transverse vibrations}

The strong magnetic field breaks the rotational symmetry for the molecular axis and, instead of rotations of the fieldfree case we have oscillations in the two-dimensional plane of the $\mathbf{R}_{\perp}$ vector. ${ }^{3}$ The degeneracy in the azimuthal angle $\phi$

\footnotetext{
${ }^{3}$ Strictly speaking, the transverse vibration and the aligned vibration are coupled, and they are governed by the two-dimensional potential $U\left(Z, R_{\perp}\right)$. Since the transverse vibrational excitation energy is larger than the aligned vibrational excitation, the time scale for the protons to adjust their $Z$ positions is much longer than the time scale for oscillations with $R_{\perp} \neq 0$ and we can consider transverse vibrations with fixed values of $Z$. However, since $\delta Z \ll Z$, an approximate separation is possible with $Z$ replaced by $Z_{0}$ for the transverse vibrations.
}

is still retained. To study the transverse vibration spectrum, we need to estimate the order of magnitude of the excess potential $\delta U\left(R_{\perp}\right) \equiv U\left(Z_{0}, R_{\perp}\right)-U\left(Z_{0}, 0\right)$.

As mentioned before, the factor $l$ in the expression $l / Z_{0}$ for the dissociation energy $D$ [Eq. (2.5)] comes from a Coulomb integral over the electron charge distribution. This integral is of the form $\ln \left(L_{z} / \hat{\rho}\right)$, where $\hat{\rho}=b^{-1 / 2}$ is the typical size of the electron wave function perpendicular to the field for $R_{\perp}=0$. When the protons are displaced by $R_{\perp}$ from the electron distribution axis, the Coulomb integral can be approximately obtained by replacing $\hat{\rho}$ with $\left(\hat{\rho}^{2}+R_{\perp}^{2}\right)^{1 / 2}$. Our order of magnitude expression for $\delta U$ is then

$\delta U\left(R_{\perp}\right) \sim \frac{1}{2 Z_{0}} \ln \left(1+\hat{\rho}^{-2} R_{\perp}^{2}\right) \sim \xi^{-1} l \ln \left(1+b R_{\perp}^{2}\right)$.

Equation (2.9) holds for any $R_{\perp} \ll Z_{0} \sim \xi l^{-1}$, but it can be approximated by a quadratic expression for the smallamplitude case of $R_{\perp} \geqslant \hat{\rho}=b^{-1 / 2} \ll Z_{0}$. In this approximation we have $\delta U \sim \xi^{-1} l b R_{\perp}^{2}$. The energy quanta for the smallamplitude transverse vibration is then

$$
\hbar \omega_{\perp 0} \sim\left(\xi^{-1} l b\right)^{1 / 2} \mu^{-1 / 2}
$$

where the subscript 0 indicates that we are at the moment neglecting the magnetic forces on the protons which, in the absence of Coulomb forces, lead to the cyclotron motions of the protons. Note that $\hbar \omega_{\perp 0}$ in Eq. (2.10) increases as $b^{1 / 2}$ with increasing field strength, faster than the logarithmic behavior of $\hbar \omega_{\|}$and $D$, but slower than the linear behavior of the cyclotron energy. For sufficiently large $b \gg 1$ we have $\hbar \omega_{\perp 0} \gg \hbar \omega_{\|}$. However, the quadratic harmonic oscillator approximation is valid only for $R_{\perp}^{2}$ up to $\sim \hat{\rho}^{2}=b^{-1}$, i.e., for $\delta U$ only up to $\delta U_{h o} \sim \xi^{-1} l$, which is less than the maximum possible potential $\Delta U_{\max } \sim D \sim \xi^{-1} l^{2}$. The number of harmonic oscillation levels in the quadratic regime is then

$$
n_{\perp h o} \sim \frac{\delta U_{h o}}{\hbar \omega_{\perp 0}} \sim \xi^{-1 / 2}\left(\frac{\mu l}{b}\right)^{1 / 2} \sim\left(\frac{b_{c r i t}}{b}\right)^{1 / 2}
$$

The degeneracy of the $n_{\perp}$ th harmonic oscillation level is $n_{\perp}$. For $n_{\perp h o} \gg 1$, the statistical weight of all harmonic oscillation levels is of order $\left(n_{\perp h o}\right)^{2}$. If we neglect the difference between $\xi$ and unity (and between $\mu$ and $m_{p} / m_{e}$ ), we see that $n_{\perp h o}$ would be less than unity when $B \gtrsim B_{c r i t}$, where $B_{\text {crit }}$ is defined in Eq. (1.5).

We now consider large amplitude transverse oscillations assuming that the magnetic force on the proton can be neglected. For a transverse oscillation wave function where the maximum value $R_{\max }$ of $R_{\perp}$ (the outer classical turning point) satisfies $\hat{\rho} \gtrsim R_{\text {max }} \gtrsim Z_{0}$, we must use the logarithmic form of Eq. (2.9) for the potential $\delta U\left(R_{\perp}\right)$. The energy level spacing decreases with increasing $R_{\text {max }}$. We can calculate the number of nodes $n_{\perp}\left(R_{\max }\right)$ of the wave function as a function of $R_{\text {max }}$ from a WKB integral of the wave number $k\left(R_{\perp}\right)$ over $d R_{\perp}$. Since we only need an order of magnitude estimate, we replace the integral by $k\left(R_{\text {max }}\right) R_{\text {max }}$, where $k\left(R_{\perp}\right) \sim\left[\mu \delta U\left(R_{\perp}\right)\right]^{1 / 2}$. Using Eq. (2.9) this gives 


$$
n_{\perp}\left(R_{\max }\right) \sim\left[\mu \xi^{-1} l \ln \left(1+b R_{\max }^{2}\right)\right]^{1 / 2} R_{\max } .
$$

The maximum number of nodes $n_{\perp \max }$ can be obtained by substituting $Z_{0} \sim \xi / l$ for $R_{\max }$. Neglecting $\ln l$ compared with $l$ itself, we have $n_{\perp \max } \sim(\xi \mu)^{1 / 2}$, independent of field strength and the same order of magnitude as $n_{\| \max }$.

Because of the azimuthal symmetry in the twodimensional $\mathbf{R}_{\perp}$ plane, the total statistical weight of the transverse excitation levels is $\sim n_{\perp \max }^{2} \sim \xi \mu$. If $b \gg b_{\text {crit }}, n_{\perp h o}$ in Eq. (2.11) would be much less than unity and the zero-point energy $\varepsilon_{\perp z p}$, i.e., the spacing between the lowest levels, is not given by Eq. (2.10). Formally, one could use Eq. (2.9) and estimate the zero-point vibration amplitude as the value of $R_{\max }$ for which Eq. (2.12) gives $n_{\perp}=1$. This would give a zero-point energy which is less than $D$, but this expression is incorrect, since the neglect of the magnetic forces on the protons is unjustified when $B \gg B_{c r i t}$. The cyclotron energy of the proton is $\hbar \omega_{p}=\hbar e B /\left(m_{p} c\right)=\left(m_{e} / m_{p}\right) b$ (a.u.). The ratio $\omega_{p} / \omega_{\perp 0}$ is of order $\left(b m_{e} / l m_{p}\right)^{1 / 2}=\left(b / b_{c r i t}\right)^{1 / 2}$ (omitting the factor $\xi)$. When $\hbar \omega_{p}$ is much larger than $\hbar \omega_{\perp 0}$, the magnetic forces on the protons are important. We will return to this subtle issue in Sec. V.

\section{METHODS FOR CALCULATING THE INTERATOMIC POTENTIAL}

In the Born-Oppenheimer approximation, the interatomic potential $U\left(Z, R_{\perp}\right)$ is given by the total electronic energy $E\left(Z, R_{\perp}\right)$ of the system when the relative positions of the protons are $Z$ along the field direction and $R_{\perp}$ perpendicular to it. Once $E\left(Z, R_{\perp}\right)$ is obtained, the electronic equilibrium state can also be determined by locating the minimum of the $E(Z, 0)$ curve.

\section{A. The aligned case: $R_{\perp}=0$}

Our method for calculating $E(Z, 0)$ is the same as in paper I. It can also be used to obtain the energy curves for the excited electronic states. Here we summarize and extend our method to take account of "configuration interaction" in $\mathrm{H}_{2}$ more accurately.

\section{1. $\mathrm{H}_{2}{ }^{+}$molecular ion}

For $\mathrm{H}_{2}{ }^{+}$, the Hamiltonian for the electron is

$$
H_{0}=H_{B}-\frac{\hbar^{2}}{2 m_{e}} \frac{\partial^{2}}{\partial z^{2}}-\frac{e^{2}}{r_{A}}-\frac{e^{2}}{r_{B}},
$$

where $r_{A}$ and $r_{B}$ are the distances between the electron and the two fixed protons, located at $z= \pm Z / 2$ along the $z$ axis. In Eq. (3.1), $H_{B}$ is the magnetic part of the Hamiltonian

$$
H_{B}=\frac{1}{2 m_{e}}\left(\mathbf{p}_{\perp}+\frac{e}{c} \mathbf{A}\right)^{2}+\frac{e}{m_{e} c} \mathbf{B} \cdot \mathbf{S},
$$

where $\mathbf{A}=\mathbf{B} \times \mathbf{r} / 2$ and $\mathbf{S}$ is the electron spin operator. Note that for electrons in the ground Landau level, we have

$$
H_{B}\left[W_{m}\left(\mathbf{r}_{\perp}\right) \chi(\downarrow)\right]=0,
$$

where $\chi(\downarrow)$ is the electron spinor with the spin aligned in the $-z$ direction (antiparallel to the field). Thus we can set
$H_{B}=0$. With the electron wave function given by $\Phi_{m 0}(\mathbf{r})=W_{m}\left(\mathbf{r}_{\perp}\right) f_{m 0}(z)$, we average over the transverse direction and obtain a one-dimensional Schrödinger equation

$$
-\frac{\hbar^{2}}{2 m_{e} \hat{\boldsymbol{\rho}}^{2}} \frac{d^{2}}{d z^{2}} f_{m 0}-\frac{e^{2}}{\hat{\rho}} \tilde{V}_{m}(z) f_{m 0}=\boldsymbol{\varepsilon}_{m 0} f_{m 0} .
$$

Here the averaged potential is given by

$$
\begin{aligned}
\tilde{V}_{m}(z) & =\int d^{2} \mathbf{r}_{\perp}\left|W_{m}\left(\mathbf{r}_{\perp}\right)\right|^{2}\left(\frac{1}{r_{A}}+\frac{1}{r_{B}}\right) \\
& =V_{m}\left(z-\frac{Z}{2}\right)+V_{m}\left(z+\frac{Z}{2}\right),
\end{aligned}
$$

where

$$
V_{m}(z) \equiv \int d^{2} \mathbf{r}_{\perp}\left|W_{m}\left(\mathbf{r}_{\perp}\right)\right|^{2} \frac{1}{r},
$$

which can be evaluated numerically (paper I). In Eqs. (3.4)(3.6) and henceforth we employ $\hat{\rho}$ as the length unit in all wave functions and average potentials (except otherwise noted). We solve the eigenvalue $\varepsilon_{m 0}$ by integrating Eq. (3.4) numerically from $z=+\infty$ to $z=0$ subject to appropriate boundary conditions (paper I). The total electronic energy is then given by

$$
E(Z, 0)=\varepsilon_{m 0}+\frac{e^{2}}{Z}
$$

Clearly, $m=0$ is the ground state, while $m=1,2, \ldots$ are the excited electronic states. We also note that the excited state of $\mathrm{H}_{2}^{+}$in which the electron occupies the $\nu>0$ orbital is not bound relative to the free atom in the ground state.

\section{2. $\mathrm{H}_{2}$ molecule: Tightly bound states $(m, v)=\left(m_{1}, 0\right),\left(m_{2}, 0\right)$}

For $\mathrm{H}_{2}$, we use the Hartree-Fock (HF) method to take account of the interaction between the electrons. The Hamiltonian of the system is

$$
H=H_{0}(1)+H_{0}(2)+\frac{e^{2}}{r_{12}}+\frac{e^{2}}{Z},
$$

where $H_{0}$ is given by Eq. (3.1) and $r_{12} \equiv\left|\mathbf{r}_{1}-\mathbf{r}_{2}\right|$. For the $\left(m_{1}, m_{2}\right)$ electronic state $\left(m_{1} \neq m_{2}\right)$, the two basis wave functions (orbitals) for the electrons are

$$
\begin{aligned}
& \Phi_{m_{1} 0}(\mathbf{r})=W_{m_{1}}\left(\mathbf{r}_{\perp}\right) f_{m_{1} 0}(z), \\
& \Phi_{m_{2} 0}(\mathbf{r})=W_{m_{2}}\left(\mathbf{r}_{\perp}\right) f_{m_{2} 0}(z) .
\end{aligned}
$$

The two-electron wave function is then given by

$$
\Psi\left(\mathbf{r}_{1}, \mathbf{r}_{2}\right)=\frac{1}{\sqrt{2}}\left[\Phi_{m_{1} 0}\left(\mathbf{r}_{1}\right) \Phi_{m_{2} 0}\left(\mathbf{r}_{2}\right)-\Phi_{m_{1} 0}\left(\mathbf{r}_{2}\right) \Phi_{m_{2} 0}\left(\mathbf{r}_{1}\right)\right] .
$$

After averaging over the transverse direction, the standard $\mathrm{HF}$ equations reduce to a set of one-dimensional equations for $f_{m_{1} 0}$ and $f_{m_{2} 0}$ : 


$$
\begin{gathered}
{\left[-\frac{\hbar^{2}}{2 m_{e} \hat{\rho}^{2}} \frac{d^{2}}{d z^{2}}-\frac{e^{2}}{\hat{\rho}} \tilde{V}_{m}(z)+\frac{e^{2}}{\hat{\rho}} K_{m}(z)-\varepsilon_{m}\right] f_{m 0}(z)} \\
\quad=\frac{e^{2}}{\hat{\rho}} J_{m}(z), \quad m=m_{1}, m_{2},
\end{gathered}
$$

where $\tilde{V}_{m}$ is given by Eq. (3.5); the direct and exchange potentials $K$ and $J$ are given by

$$
\begin{gathered}
K_{m_{1}}(z)=\int d z^{\prime} f_{m_{2} 0}\left(z^{\prime}\right)^{2} D_{m_{1} m_{2}}\left(z-z^{\prime}\right), \\
J_{m_{1}}(z)=f_{m_{2} 0}(z) \int d z^{\prime} f_{m_{1} 0}\left(z^{\prime}\right) f_{m_{2} 0}\left(z^{\prime}\right) E_{m_{1} m_{2}}\left(z-z^{\prime}\right),
\end{gathered}
$$

and similarly for $K_{m_{2}}$ and $J_{m_{2}}$. In Eqs. (3.13) and (3.14), $D_{m_{1} m_{2}}$ and $E_{m_{1} m_{2}}$ are the direct and exchange interaction kernels defined by

$$
\begin{aligned}
D_{m_{1} m_{2}}\left(z_{1}-z_{2}\right)= & \int d^{2} \mathbf{r}_{1 \perp} d^{2} \mathbf{r}_{2 \perp}\left|W_{m_{1}}\left(\mathbf{r}_{1 \perp}\right)\right|^{2}\left|W_{m_{2}}\left(\mathbf{r}_{2 \perp}\right)\right|^{2} \\
& \times \frac{1}{r_{12}}, \\
E_{m_{1} m_{2}}\left(z_{1}-z_{2}\right)= & \int d^{2} \mathbf{r}_{1 \perp} d^{2} \mathbf{r}_{2 \perp} W_{m_{1}}\left(\mathbf{r}_{1 \perp}\right) W_{m_{2}}\left(\mathbf{r}_{2 \perp}\right) \\
& \times W_{m_{1}}^{*}\left(\mathbf{r}_{2 \perp}\right) W_{m_{2}}^{*}\left(\mathbf{r}_{1 \perp}\right) \frac{1}{r_{12}}
\end{aligned}
$$

The functions $D_{m_{1} m_{2}}(z)$ and $E_{m_{1} m_{2}}(z)$ are related to the Coulomb interaction potential $V_{m}$ [Eq. (3.6)] by

$$
\begin{aligned}
& D_{m_{1} m_{2}}(z)=\sum_{s=0}^{m_{1}+m_{2}} d_{s}\left(m_{1}, m_{2}\right) \frac{1}{\sqrt{2}} V_{s}\left(\frac{z}{\sqrt{2}}\right), \\
& E_{m_{1} m_{2}}(z)=\sum_{s=0}^{m_{1}+m_{2}} e_{s}\left(m_{1}, m_{2}\right) \frac{1}{\sqrt{2}} V_{s}\left(\frac{z}{\sqrt{2}}\right),
\end{aligned}
$$

where the coefficients $d_{s}$ and $e_{s}$ are given in paper I. We solve Eq. (3.12) numerically using a shooting algorithm (for details, see paper I). Once the wave function $f_{m 0}(z)$ and the eigenvalues $\varepsilon_{m 0}$ are obtained, the total electronic energy of the system is calculated via

$$
\begin{aligned}
E= & \langle\Psi|H| \Psi\rangle \\
= & \frac{e^{2}}{Z}+\varepsilon_{m_{1} 0}+\varepsilon_{m_{2} 0}-\frac{e^{2}}{\hat{\rho}} \int d z_{1} d z_{2} f_{m_{1} 0}\left(z_{1}\right)^{2} f_{m_{2} 0}\left(z_{2}\right)^{2} \\
& \times D_{m_{1} m_{2}}\left(z_{1}-z_{2}\right)+\frac{e^{2}}{\hat{\rho}} \int d z_{1} d z_{2} f_{m_{1} 0}\left(z_{1}\right) f_{m_{2} 0}\left(z_{2}\right) \\
& \times f_{m_{1} 0}\left(z_{2}\right) f_{m_{2} 0}\left(z_{1}\right) E_{m_{1} m_{2}}\left(z_{1}-z_{2}\right),
\end{aligned}
$$

where the fourth term on the right-hand side represents the electron direct interaction $\left(-E^{d i r}\right)$, and the fifth term the exchange interaction $\left(-E^{\text {exch }}\right)$.
The Hartree-Fock method discussed above can be used to obtain accurately the electronic energy near the equilibrium separation $Z_{0}$. However, as noted in paper $\mathrm{I}$, as $Z$ increases, the resulting $E(Z, 0)$ becomes less reliable. Moreover, as $Z \rightarrow \infty, E(Z, 0)$ does not approach the sum of the energies of two isolated atoms, one in the $m_{1}$ th state, another in the $m_{2}$ th state. The reason is that as $Z$ increases, a second configuration of electron orbitals becomes more and more degenerate with the first configuration in Eq. (3.11), and there must be mixing of these two different configurations. This "configuration interaction" also occurs in the zero-field $\mathrm{H}_{2}$ molecule [29]. Here the electron configuration that mixes with $\Psi_{1} \equiv \Psi \quad[$ Eq. (3.11)] is

$\Psi_{2}\left(\mathbf{r}_{1}, \mathbf{r}_{2}\right)=\frac{1}{\sqrt{2}}\left[\Phi_{m_{1} 1}\left(\mathbf{r}_{1}\right) \Phi_{m_{2} 1}\left(\mathbf{r}_{2}\right)-\Phi_{m_{1} 1}\left(\mathbf{r}_{2}\right) \Phi_{m_{2} 1}\left(\mathbf{r}_{1}\right)\right]$,

which is the same as $\Psi_{1}$ except $\nu=1$ in the electron orbitals. Both $\Psi_{1}$ and $\Psi_{2}$ have the same symmetry with respect to the Hamiltonian in Eq. (3.8): the total angular momentum along the $z$ axis is $M_{L z}=1$, the total electron spin is $M_{S z}=-1$, and both $\Psi_{1}$ and $\Psi_{2}$ are even with respect to the operation $\mathbf{r}_{i} \rightarrow-\mathbf{r}_{i}$. As a result, the matrix element $\left\langle\Psi_{1}|H| \Psi_{2}\right\rangle$ is nonzero.

To take account of the mixing of these two configurations, we need to extend the standard HF method involving one configuration to $H F$ with multiconfigurations (HFMC). This is done as follows. We calculate the energies and wave functions for both $\Psi_{1}$ and $\Psi_{2}$ using the HF equations [Eq. (3.12) with $\nu=0$ and $\nu=1]$. The matrix elements $H_{i j}(i, j=1,2)$ are then calculated: $H_{11}=\left\langle\Psi_{1}|H| \Psi_{1}\right\rangle$ is given by Eq. (3.19) and the expression for $H_{22}=\left\langle\Psi_{2}|H| \Psi_{2}\right\rangle$ is similar. The mixing matrix element is given by

$$
\begin{aligned}
H_{12}= & \left\langle\Psi_{1}|H| \Psi_{2}\right\rangle \\
= & \frac{e^{2}}{\hat{\rho}} \int d z_{1} d z_{2} f_{m_{1} 0}\left(z_{1}\right) f_{m_{1} 1}\left(z_{1}\right) f_{m_{2} 0}\left(z_{2}\right) f_{m_{2} 1}\left(z_{2}\right) \\
& \times D_{m_{1} m_{2}}\left(z_{1}-z_{2}\right)-\frac{e^{2}}{\hat{\rho}} \int d z_{1} d z_{2} f_{m_{1} 0}\left(z_{1}\right) f_{m_{2} 1}\left(z_{1}\right) \\
& \times f_{m_{2} 0}\left(z_{2}\right) f_{m_{1} 1}\left(z_{2}\right) E_{m_{1} m_{2}}\left(z_{1}-z_{2}\right) .
\end{aligned}
$$

The total electronic energy in this HFMC scheme is obtained by solving the secular equation $\operatorname{det}\left|H_{i j}-E \delta_{i j}\right|=0$, which yields, for the lowest energy state,

$E=\frac{1}{2}\left(H_{11}+H_{22}\right)-\frac{1}{2}\left[\left(H_{11}-H_{22}\right)^{2}+4 H_{12}^{2}\right]^{1 / 2}$.

In Fig. 1, we show the electronic energy curves of $\mathrm{H}_{2}$ at $B_{12}=1$, obtained using our HFMC method. The tightly bound electronic states are $\left(m_{1}, m_{2}\right)=(0,1),(0,2)$, and $(0,3)$. These are the only states for which the minimum in the energy curves is less than the energy $2 E_{a}=-323 \mathrm{eV}$ of two isolated atoms in the ground state. Notice that as $Z$ increases, the molecular electronic energy becomes larger than $2 E_{a}$, reflecting the fact that, in a superstrong magnetic field, forming such a tightly bound molecule requires first activating one of the atoms to an excited state. However, as $Z$ 


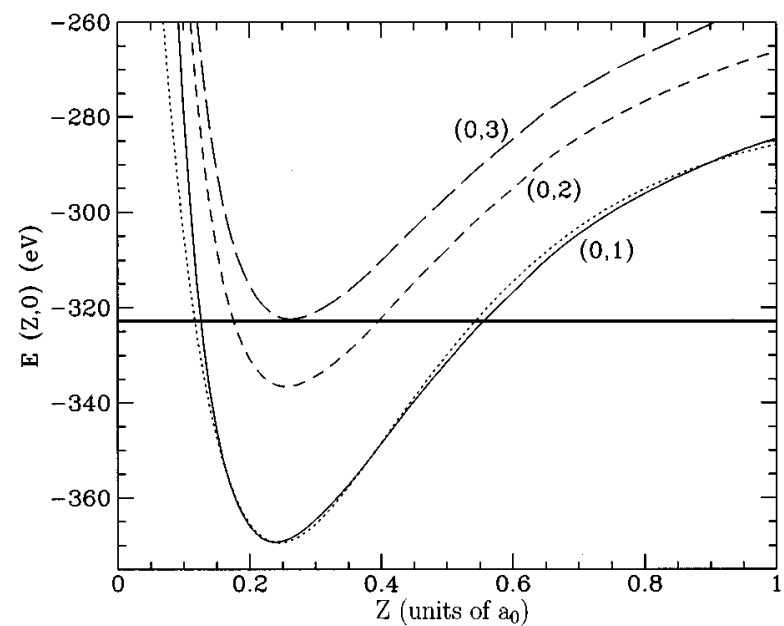

FIG. 1. The electronic energy curves $E(Z, 0)$ for the tightly bound states of $\mathrm{H}_{2}$ molecule at $B=10^{12} \mathrm{G}$ when the molecular axis is aligned with the magnetic field axis. The electrons occupy the $(m, \nu)=\left(m_{1}, 0\right)$ and $\left(m_{2}, 0\right)$ orbitals $\left(m_{1} \neq m_{2}\right)$. The solid line is for the state $\left(m_{1}, m_{2}\right)=(0,1)$, the short-dashed line for $(0,2)$, the longdashed line for $(0,3)$. The dotted line is from the fitting using the Morse potential [Eq. (4.2)]. The dark solid line corresponds to the energy of two isolated $\mathrm{H}$ atoms in the ground state $2 E_{a}=-323 \mathrm{eV}$.

increases, the energy of the $\left(m_{1}, m_{2}\right)$ state does approach $E_{a}\left(m_{1}\right)+E_{a}\left(m_{2}\right)$. Near the equilibrium separation, the energy obtained using HFMC agrees well with that of the standard HF (the difference is less than 1\%). Thus the standard $\mathrm{HF}$ is adequate for determining the equilibrium electronic energy of the molecule. However, the HFMC method is crucial to obtaining the correct large $Z$ behavior of $E(Z, 0)$, therefore the aligned vibrational energy levels of the molecule (Sec. IV B).

\section{3. $\mathrm{H}_{2}$ molecule: Weakly bound state $(m, \nu)=(0,0),(0,1)$}

These states can be similarly calculated using the HF method. Instead of Eqs. (3.9) and (3.10), the electron orbitals are $\Phi_{00}$ and $\Phi_{01}$. Figure 2 shows an example of the energy curve at $B_{12}=1$. Clearly, the $E(Z, 0)$ curve of such a state is much shallower than those of the tightly bound states discussed in Sec. III A 2. In the limit of $Z \rightarrow \infty$, the energy curve approaches $2 E_{a}$, i.e., no "activation energy" is needed to form a molecule in the weakly bound state.

\section{B. General molecular axis orientation: $\boldsymbol{R}_{\perp} \neq \mathbf{0}$}

Unlike the case of Sec. III A when the molecular axis coincides with the magnetic-field direction, where we can obtain the interatomic potential $E(Z, 0)$ with great accuracy, in the case when the molecular axis deviates from the magnetic-field direction, the electronic energy $E\left(Z, R_{\perp}\right)$ is much harder to calculate. This is because the azimuthal symmetry of the transverse wave function of an electron is broken. Although the electrons still stay in the ground Landau level, $m$ in the Landau wave function $W_{m}\left(\mathbf{r}_{\perp}\right)$ [Eq. (2.2)] is no longer a good quantum number, and the transverse wave function of an electron must involve mixing of different $m$ states. Nevertheless, we can still obtain a reasonable upper limit for the interatomic potential curve $E\left(Z, R_{\perp}\right)$, and hence

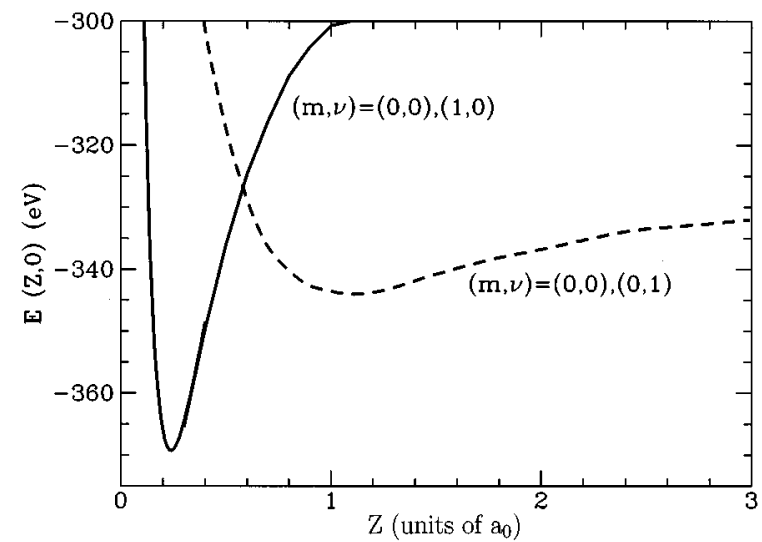

FIG. 2. The electronic energy curves $E(Z, 0)$ of $\mathrm{H}_{2}$ molecule at $B=10^{12} \mathrm{G}$ when the molecular axis is aligned with the magnetic field axis. The solid line corresponds to the tightly bound state in which the electrons occupy the $(m, \nu)=(0,0)$ and $(1,0)$ orbitals, the dashed line corresponds to the weakly bound state in which the electrons occupy the $(0,0)$ and $(0,1)$ orbitals.

an upper limit for the transverse vibrational excitation energy quanta $\hbar \omega_{\perp 0}$. We consider two ansatzes, appropriate for small $R_{\perp}$, and large $R_{\perp}$, respectively.

\section{Ansatz A}

Suppose the two protons are located at $\left( \pm R_{\perp} / 2,0, \pm Z / 2\right)$ in a rectangular coordinate system. For sufficiently small $R_{\perp}$, the transverse wave function is expected to be close to $W_{m}\left(\mathbf{r}_{\perp}\right)$. Thus we assume the electron wave function in $\mathrm{H}_{2}{ }^{+}$is given by $\Phi_{m 0}(\mathbf{r})=W_{m}\left(\mathbf{r}_{\perp}\right) f_{m 0}(z)$. The equation for $f_{m 0}$ is the same as Eq. (3.4), except that the potential $\tilde{V}_{m}(z)$ is replaced by

$$
\tilde{V}_{m m}\left(z, R_{\perp} / 2\right)=V_{m m}\left(\left|z-\frac{Z}{2}\right|, \frac{R_{\perp}}{2}\right)+V_{m m}\left(\left|z+\frac{Z}{2}\right|, \frac{R_{\perp}}{2}\right),
$$

where

$$
\begin{aligned}
V_{m m}\left(z, R_{\perp} / 2\right) \equiv & \int d^{2} \mathbf{r}_{\perp}\left|W_{m}\left(\mathbf{r}_{\perp}\right)\right|^{2} \frac{1}{\left|\mathbf{r}-\mathbf{R}_{\perp} / 2\right|} \\
= & \int_{0}^{\infty} d q \exp \left(-\frac{1}{2} q^{2}-q|z|\right) \\
& \times J_{0}\left(\frac{q R_{\perp}}{2}\right) L_{m}\left(\frac{1}{2} q^{2}\right)
\end{aligned}
$$

(see Appendix A). Here $J_{0}$ is the Bessel function of zeroth order and $L_{m}$ is the Laguerre polynormial of order $m$ [30]. We use a standard quadrature algorithm (e.g., [31]) to evaluate Eq. (3.24). The Schrödinger equation similar to Eq. (3.4) can be solved to determine the eigenvalue $\varepsilon_{m 0}\left(Z, R_{\perp}\right)$, and the total electronic energy is then given by

$$
E_{m 0}\left(Z, R_{\perp}\right)=\varepsilon_{m 0}\left(Z, R_{\perp}\right)+\frac{e^{2}}{\left(Z^{2}+R_{\perp}^{2}\right)^{1 / 2}} .
$$


As noted before, in this general situation, $m$ is not a good quantum number, but we nevertheless use it to distinguish different electronic states.

In this ansatz, the equations for $\mathrm{H}_{2}$ are also similar to those in Sec. III A. We still assume the electron orbitals to be given by Eqs. (3.9) and (3.10). The HF equations (3.12)(3.14) remain valid except the ion-electron interaction potential $\tilde{V}_{m}(z)$ is replaced by $\tilde{V}_{m m}\left(z, R_{\perp} / 2\right)$. The electronelectron interaction kernels are unchanged. The total electronic energy is still given by Eq. (3.19) with $e^{2} / Z$ replaced by $e^{2} /\left(Z^{2}+R_{\perp}^{2}\right)^{1 / 2}$.

We now estimate the regime of validity of this ansatz. As an example, let us consider the ground electronic state of $\mathrm{H}_{2}{ }^{+}$. In general, the transverse wave function of the electron is a superposition of different Landau ground-state wave functions, i.e.,

$$
\Phi_{\perp}\left(\mathbf{r}_{\perp}\right)=\sum_{m} A_{m} W_{m}\left(\mathbf{r}_{\perp}\right)
$$

and $\Phi(\mathbf{r})=\Phi_{\perp}\left(\mathbf{r}_{\perp}\right) f(z)$ is the total wave function (see also Appendix B). For simplicity, just consider the first two terms in the expansion (3.26), i.e., $\Phi_{\perp}\left(\mathbf{r}_{\perp}\right)=A_{0} W_{0}\left(\mathbf{r}_{\perp}\right)$ $+A_{1} W_{1}\left(\mathbf{r}_{\perp}\right)$, with $\left|A_{1}\right| \ll\left|A_{0}\right|$ for the ground state. Substituting $\Phi(\mathbf{r})$ into the Schrödinger equation and average over $\mathbf{r}_{\perp}$, we obtain (in atomic units)

$$
\begin{aligned}
& -\frac{1}{2} \frac{d^{2}}{d z^{2}} f-\tilde{V}_{00}(z) f+\frac{A_{1}}{A_{0}} \tilde{V}_{01}(z) f=\varepsilon f, \\
& -\frac{1}{2} \frac{d^{2}}{d z^{2}} f-\tilde{V}_{11}(z) f+\frac{A_{0}}{A_{1}} \tilde{V}_{10}(z) f=\varepsilon f,
\end{aligned}
$$

where $\tilde{V}_{m m^{\prime}}$ is defined similar to Eq. (3.24). Since $\left|\tilde{V}_{01}\right| \ll\left|\tilde{V}_{00}\right|$ and $\left|\tilde{V}_{01}\right| \ll\left|\tilde{V}_{11}\right|$, from Eqs. (3.27) and (3.28) we have $A_{1} / A_{0} \simeq \tilde{V}_{10} /\left(\tilde{V}_{11}-\tilde{V}_{00}\right)$. Substituting this into Eq. (3.27), we have

$$
-\frac{1}{2} \frac{d^{2}}{d z^{2}} f-\tilde{V}_{00} f+\frac{\tilde{V}_{01} \tilde{V}_{10}}{\tilde{V}_{11}-\tilde{V}_{00}} f=\varepsilon f .
$$

Comparing with the zeroth order eigenvalue $\varepsilon_{m}^{(0)}$ (which does not take into account the mixing), the corrected eigenvalue for the ground state is then given by

$\varepsilon_{0} \simeq \varepsilon_{0}^{(0)}+\left\langle\frac{\tilde{V}_{01} \tilde{V}_{10}}{\tilde{V}_{11}-\tilde{V}_{00}}\right\rangle \sim \varepsilon_{0}^{(0)}+\left\langle\frac{\tilde{V}_{01} \tilde{V}_{10}}{\varepsilon_{0}^{(0)}-\varepsilon_{1}^{(0)}}\right\rangle$,

where $\langle\cdots\rangle$ denotes expectation value. Requiring the second term to be smaller than the first, we have $\left\langle\tilde{V}_{01}\right\rangle^{2} / l \ll l^{2}$, where we have used $\left|\varepsilon_{0}^{(0)}\right| \sim l^{2}$ and $\left|\varepsilon_{0}^{(0)}-\varepsilon_{1}^{(0)}\right| \sim l$. Since

$$
\begin{aligned}
\left\langle\tilde{V}_{01}\right\rangle & \sim-\left\langle\mathbf{R}_{\perp} \cdot \vec{\nabla} \frac{1}{r}\right\rangle_{01} \sim-R_{\perp}\left\langle\frac{x}{r^{3}}\right\rangle_{01} \sim-R_{\perp} \hat{\boldsymbol{\rho}}\left\langle\frac{1}{r^{3}}\right\rangle \\
& \sim-\frac{1}{L_{z} \hat{\rho}} R_{\perp}
\end{aligned}
$$

the condition for the ansatz to be valid is $R_{\perp} \ll l^{1 / 2} \hat{\rho}$, i.e., the proton transverse displacement must be smaller than $\sim \hat{\rho}$.

\section{Ansatz B}

At large $R_{\perp}$, the molecule should become two individual atoms (or atom plus ion). Here we set up a rectangular coordinate system so that the two protons are located at $(0,0, Z / 2)$ and $\left(R_{\perp}, 0,-Z / 2\right)$. The electron wave function of $\mathrm{H}_{2}^{+}$is assumed to be $\Phi_{m 0}(\mathbf{r})=W_{m}\left(\mathbf{r}_{\perp}\right) f_{m 0}(z)$, i.e., the electron cloud is centered on one of the protons. Then the problem is essentially equivalent to calculating how an atom is affected by an external ion. The Schrödinger equation (3.4) still applies except that the potential $\tilde{V}_{m}(z)$ is replaced by

$\tilde{V}_{m m}^{\prime}\left(z, R_{\perp}\right)=V_{m}\left(\left|z-\frac{Z}{2}\right|\right)+V_{m m}\left(\left|z+\frac{Z}{2}\right|, R_{\perp}\right)$,

where the functions $V_{m}$ and $V_{m m}$ are defined in Eqs. (3.6) and (3.24), respectively. The eigenvalue can again be solved and thus the total energy $E\left(Z, R_{\perp}\right)$ can be obtained.

In this ansatz, since the electron wave function is not symmetric with respect to $z \rightarrow-z$, the numerical method used in Secs. III A and III B 1 (see paper I) needs modification. Here we integrate the equation from both $\infty$ and $-\infty$. The eigenvalue is obtained by matching the solution at $z=0$ (see [31]). We also note that the classical quadrupole formula for the ion-atom interaction is not applicable here, since we always consider $R_{\perp} \lesssim L_{z}$ for a bound state.

For $\mathrm{H}_{2}$, we choose the two electron orbitals centered on each of the protons:

$$
\begin{gathered}
\Phi_{m_{1}}(\mathbf{r})=W_{m_{1}}\left(\mathbf{r}_{\perp}\right) f_{m_{1} 0}(z), \\
\Phi_{m_{2}}(\mathbf{r})=W_{m_{2}}\left(\mathbf{r}_{\perp}-\mathbf{R}_{\perp}\right) f_{m_{2} 0}(z) e^{-i B R_{\perp} y / 2} .
\end{gathered}
$$

The extra factor $e^{-i B R_{\perp} y / 2}$ in $\Phi_{m_{2}}(\mathbf{r})$ comes from a gauge transformation, so that the displaced Landau wave function $W_{m_{2}}\left(\mathbf{r}_{\perp}-\mathbf{R}_{\perp}\right)$ is still an eigenstate of the magnetic Hamiltonian with a fixed gauge [Eq. (3.2)], i.e.,

$$
H_{B}\left[W_{m_{2}}\left(\mathbf{r}_{\perp}-\mathbf{R}_{\perp}\right) e^{-i B R_{\perp} y / 2} \chi(\downarrow)\right]=0 .
$$

With this ansatz for the basis wave functions, the HF equations given in Sec. III A [Eq. (3.12)] can be applied, except that $\tilde{V}_{m}(z)$ must be replaced by $\tilde{V}_{m m}^{\prime}\left(z, R_{\perp}\right)$ given in Eq. (3.32). Also, the direct and exchange kernels [Eqs. (3.15) and (3.16)] are replaced by 


$$
\begin{gathered}
\tilde{D}_{m_{1} m_{2}}\left(z_{1}-z_{2}, R_{\perp}\right)=\int d^{2} \mathbf{r}_{1 \perp} d^{2} \mathbf{r}_{2 \perp}\left|W_{m_{1}}\left(\mathbf{r}_{1 \perp}\right)\right|^{2}\left|W_{m_{2}}\left(\mathbf{r}_{2 \perp}-\mathbf{R}_{\perp}\right)\right|^{2} \frac{1}{r_{12}} \\
\tilde{E}_{m_{1} m_{2}}\left(z_{1}-z_{2}, R_{\perp}\right)=\int d^{2} \mathbf{r}_{1 \perp} d^{2} \mathbf{r}_{2 \perp} W_{m_{1}}\left(\mathbf{r}_{1 \perp}\right) W_{m_{2}}\left(\mathbf{r}_{2 \perp}-\mathbf{R}_{\perp}\right) W_{m_{1}}^{*}\left(\mathbf{r}_{2 \perp}\right) W_{m_{2}}^{*}\left(\mathbf{r}_{1 \perp}-\mathbf{R}_{\perp}\right) \times e^{i B R_{\perp}\left(y_{1}-y_{2}\right) / 2} \frac{1}{r_{12}} .
\end{gathered}
$$

The function $\tilde{D}_{m_{1} m_{2}}\left(z, R_{\perp}\right)$ can be expressed as a sum of the function $V_{m m}$ (see Appendix A),

$$
\tilde{D}_{m_{1} m_{2}}\left(z, R_{\perp}\right)=\sum_{s=0}^{m_{1}+m_{2}} d_{s}\left(m_{1}, m_{2}\right) \frac{1}{\sqrt{2}} V_{s s}\left(\frac{z}{\sqrt{2}}, \frac{R_{\perp}}{\sqrt{2}}\right),
$$

thus it can be evaluated using Eq. (3.24). For $R_{\perp} \gg \hat{\rho}$, the exchange interaction between electrons can be neglected since the electron clouds are separated, i.e., we can set $\tilde{E}_{m_{1} m_{2}}\left(z, R_{\perp}\right)=0$. Therefore, we only need to solve the corresponding Hartree equations:

$$
\begin{aligned}
& {\left[-\frac{\hbar^{2}}{2 m_{e} \hat{\rho}^{2}} \frac{d^{2}}{d z^{2}}-\frac{e^{2}}{\hat{\rho}} \tilde{V}_{m m}^{\prime}\left(z, R_{\perp}\right)+\frac{e^{2}}{\hat{\rho}} \tilde{K}_{m}\left(z, R_{\perp}\right)-\varepsilon_{m}\right] f_{m 0}(z)} \\
& \quad=0, \quad m=m_{1}, m_{2},
\end{aligned}
$$

where $\tilde{K}_{m}$ is given by

$$
\tilde{K}_{m_{1}}\left(z, R_{\perp}\right)=\int d z^{\prime} f_{m_{2} 0}\left(z^{\prime}\right)^{2} \tilde{D}_{m_{1} m_{2}}\left(z-z^{\prime}, R_{\perp}\right)
$$

and similarly for $\tilde{K}_{m_{2}}$.

In Fig. 3, we show the energy curve for $\mathrm{H}_{2}{ }^{+}$at $B_{12}=1$. The electron is assumed to be in the $m=0$ state. The electronic energy curves $E\left(Z, R_{0}\right)$ are calculated using ansatz A with a fixed value of $R_{\perp}=R_{0}$. Each curve has a minimum at

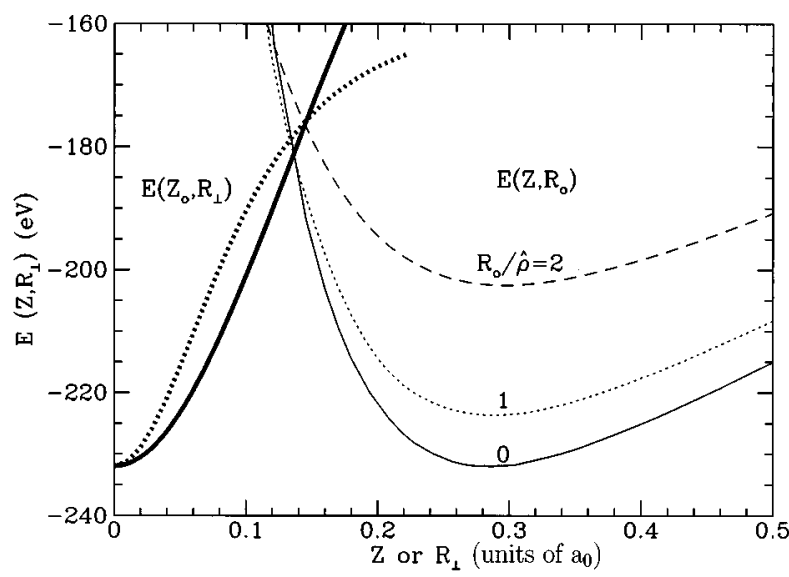

FIG. 3. The electronic energy curves for the ground state of $\mathrm{H}_{2}{ }^{+}$at $B=10^{12} \mathrm{G}$. The light lines show the $E\left(Z, R_{0}\right)$ curves with a fixed $R_{\perp}=R_{0}$ for $R_{0}=0$ (solid line), $R_{0}=\hat{\rho}$ (dotted line), and $R_{0}=2 \hat{\rho}$ (dashed line). The dark lines show the function $E\left(Z_{0}, R_{\perp}\right)$ for a fixed value of $Z_{0}$ given by the equilibrium separation of the protons. The solid line is calculated using ansatz $\mathrm{A}$, the dotted line using ansatz B (see Sec. III B).
$Z=Z_{e q}\left(R_{0}\right)$. We see that this equilibrium position is almost independent of $R_{0}$, i.e., $Z_{e q}\left(R_{0}\right) \simeq Z_{e q}(0)=Z_{0}$. The curves $E\left(Z_{0}, R_{\perp}\right)$ with a fixed value of $Z_{0}$ are calculated using both ansatzes discussed above. For $R_{\perp}$ less than a few times $\hat{\rho}$, ansatz A yields lower energy, while for larger $R_{\perp}$, ansatz $\mathrm{B}$ gives the correct behavior for the energy curve, i.e., $E\left(Z_{0}, R_{\perp}\right) \rightarrow E_{a}$ as $R_{\perp}$ increases. Similar behavior for $\mathrm{H}_{2}$ can also be obtained. It is evident from Fig. 2 that the curves $E\left(Z_{0}, R_{\perp}\right)$ are much steeper than $E\left(Z, R_{0}\right)$. Thus the molecule is tied much more "rigidly" to the magnetic-field line than along the field axis.

\section{RESULTS FOR THE MOLECULAR EXCITATION LEVELS}

In this section, we present our numerical results for the excitation levels of $\mathrm{H}_{2}$. The results for $\mathrm{H}_{2}{ }^{+}$are also included for completeness and for comparing with previous calculations (no previous results for $\mathrm{H}_{2}$ are available).

\section{A. Electronic excitations}

The equilibrium electronic state is determined by the minimum in the energy curve $E(Z, 0)$ (cf. Fig. 1). For $\mathrm{H}_{2}{ }^{+}$, the electronic state is characterized by a single quantum number $m$. For $\mathrm{H}_{2}$, there are two types of electronic excitations: the "tightly bound" levels correspond to electrons in the $(m, \nu)=\left(m_{1}, 0\right)$ and $\left(m_{2}, 0\right)$ orbitals, and the "weakly bound" excitation corresponds to $(m, \nu)=(0,0),(0,1)$. We have calculated all the electronic bound states of $\mathrm{H}_{2}^{+}$and $\mathrm{H}_{2}$ for $0.1 \leqslant B_{12} \leqslant 10$. The results for $\mathrm{H}_{2}$ are summarized in Table II (for the tightly bound states) and Table III (for the weakly bound state), while the results for $\mathrm{H}_{2}^{+}$are given in Table IV. Here, by "bound" we mean that the equilibrium electronic energy $E_{m}$ of the molecule is less than $E_{a} \equiv E_{a}(0)$, the energy of a single atom in the ground state (for $\mathrm{H}_{2}^{+}$), or $2 E_{a}$, the energy of two atoms $\left(\right.$ for $\mathrm{H}_{2}$ ). Clearly, $\mathrm{H}_{2}$ has more electronic excitation levels than $\mathrm{H}_{2}{ }^{+}$. As $B$ increases, the number of bound levels in $\mathrm{H}_{2}$ increases. For $B_{12} \leqslant 10$, only single-excitation tightly bound levels, i.e., those with $m_{1}=0$, are bound. The doubleexcitation levels, such as $(1,2)$ are not bound until the field strength increases to $B_{12} \gtrsim 50$. Excluding the zero-point oscillation energy of the protons (see Sec. V), the dissociation energy of the $\mathrm{H}_{2}$ molecule is given by $D^{(\infty)}=2 E_{a}-E_{m}$.

We have also calculated the ground-state energy of the molecule in the stronger field regime. For $B_{12} \geq 10$, our numerical results can be well fitted to the form

$$
E_{m} \simeq-0.091(\ln b)^{2.7} \quad \text { (a.u.). }
$$

A more general fitting formula for $D^{(\infty)}$ is given in Eq. (5.2). 
TABLE II. The tightly bound energy levels of a $\mathrm{H}_{2}$ molecule in which the electrons occupy the $(m, \nu)=\left(m_{1}, 0\right)$ and $\left(m_{2}, 0\right)$ orbitals $\left(m_{1} \neq m_{2}\right)$. Here $B_{12}=B /\left(10^{12} \mathrm{G}\right), E_{a}$ is the ground-state energy of the $\mathrm{H}$ atom, $m_{1}, m_{2}$ are the quantum numbers specifying the electronic excitations, $E_{m}$ is the electronic energy of the molecule, $Z_{0}$ is the equilibrium interatomic separation ( $a_{0}$ is the Bohr radius), $D_{m}$ is defined by $D_{m} \equiv E_{a}\left(m_{1}\right)+E_{a}\left(m_{2}\right)-E_{m}\left(m_{1}, m_{2}\right), \hbar \omega_{\|}$is the aligned vibrational energy quanta, and $\hbar \omega_{\perp 0}$ is the transverse vibrational energy quanta (neglecting the magnetic forces on protons).

\begin{tabular}{|c|c|c|c|c|c|c|c|}
\hline$B_{12}$ & $2 E_{a}(\mathrm{eV})$ & $m_{1}, m_{2}$ & $\overline{E_{m}(\mathrm{eV})}$ & $Z_{0}$ (units of $\left.a_{0}\right)$ & $\overline{D_{m}(\mathrm{eV})}$ & $\hbar \omega_{\|}(\mathrm{eV})$ & $\overline{\hbar \omega_{\perp 0}(\mathrm{eV})}$ \\
\hline 0.1 & -152.8 & 0,1 & -161 & 0.52 & 31.7 & 3.0 & 2.6 \\
\hline \multirow[t]{2}{*}{0.5} & -260.4 & 0,1 & -291 & 0.30 & 67.5 & 7.2 & 8.7 \\
\hline & & 0,2 & -264 & 0.32 & 55.7 & 6.3 & \\
\hline \multirow[t]{3}{*}{1} & -323.0 & 0,1 & -369 & 0.25 & 91.0 & 9.8 & 14 \\
\hline & & 0,2 & -337 & 0.26 & 76.5 & 8.8 & \\
\hline & & 0,3 & -323 & 0.26 & 73.0 & 8.3 & \\
\hline \multirow[t]{4}{*}{2} & -397.0 & 0,1 & -466 & 0.20 & 121 & 13 & 23 \\
\hline & & 0,2 & -425 & 0.21 & 103 & 12 & \\
\hline & & 0,3 & -408 & 0.21 & 98.3 & 11 & \\
\hline & & 0,4 & -398 & 0.22 & 96.8 & 11 & \\
\hline \multirow[t]{6}{*}{5} & -514.2 & 0,1 & -623 & 0.15 & 173 & 19 & 42 \\
\hline & & 0,2 & -573 & 0.16 & 150 & 18 & \\
\hline & & 0,3 & -550 & 0.16 & 143 & 17 & \\
\hline & & 0,4 & -537 & 0.16 & 142 & 16 & \\
\hline & & 0,5 & -527 & 0.17 & 141 & 16 & \\
\hline & & 0,6 & -519 & 0.17 & 140 & 16 & \\
\hline \multirow[t]{10}{*}{10} & -619.2 & 0,1 & -769 & 0.12 & 224 & 25 & 65 \\
\hline & & 0,2 & -709 & 0.13 & 196 & 23 & \\
\hline & & 0,3 & -682 & 0.13 & 188 & 22 & \\
\hline & & 0,4 & -666 & 0.14 & 185 & 22 & \\
\hline & & 0,5 & -654 & 0.14 & 183 & 21 & \\
\hline & & 0,6 & -645 & 0.14 & 183 & 21 & \\
\hline & & 0,7 & -638 & 0.14 & 182 & 21 & \\
\hline & & 0,8 & -632 & 0.14 & 182 & 20 & \\
\hline & & 0,9 & -627 & 0.14 & 182 & 20 & \\
\hline & & 0,10 & -623 & 0.14 & 181 & 20 & \\
\hline
\end{tabular}

We note that as $B$ increases, the energy $\left|E_{m}\right|$ of the tightly bound levels of $\mathrm{H}_{2}$ increases rapidly, while that of the weakly bound level does not change appreciably. For $l=\ln b \gg 1$, the weakly bound state is indeed an excited state of the $\mathrm{H}_{2}$ molecule. For $B_{12} \leq 0.2$, however, we find that the weakly bound state actually has lower energy than the tightly bound level $\left(m_{1}, m_{2}\right)=(0,1)$. Thus for such relatively small magnetic-field strength, the weakly bound state is the actual ground state of the molecule.

TABLE III. The energy of the weakly bound state of $\mathrm{H}_{2}$ in which the electrons occupy the $(m, \nu)=(0,0)$ and $(0,1)$ orbitals. Here $B_{12}=B /\left(10^{12} \mathrm{G}\right), E_{m}$ is the energy of the molecule, $D_{\nu}=\left|E_{m}\right|-2\left|E_{a}\right|$ is the dissociation energy of the level (neglecting the zero-point oscillation energy of the protons), and $Z_{0}$ is the equilibrium interatomic separation ( $a_{0}$ is the Bohr radius).

\begin{tabular}{cccc}
\hline \hline$B_{12}$ & $E_{m}(\mathrm{eV})$ & $D_{\nu}(\mathrm{eV})$ & $Z_{0}$ (units of $\left.a_{0}\right)$ \\
\hline 0.1 & -167 & 14 & 1.5 \\
0.5 & -279 & 19 & 1.3 \\
1 & -344 & 21 & 1.1 \\
2 & -421 & 24 & 0.99 \\
5 & -542 & 28 & 0.89 \\
10 & -649 & 30 & 0.72 \\
\hline \hline
\end{tabular}

\section{B. Aligned vibrational excitations}

In the standard Born-Oppenheimer approximation, the Hamiltonian describing the relative motion of the protons is simply $H_{i}=\mathbf{P}^{2} /(2 \mu)+U\left(Z, R_{\perp}\right)$, where $\mu$ is the reduced mass of the proton pair, and the interatomic interaction potential $U$ is given by the total electronic energy $E\left(Z, R_{\perp}\right)$, as calculated in Sec. III. For the vibrations along the $z$ direction, there is no magnetic force on the protons, and Eq. (4.1) is a good approximation. The aligned vibrations are governed by the potential $U(Z, 0)=E(Z, 0)$, which we can fit to a Morse potential (e.g., [28])

$$
U(Z, 0)=D_{m}\left\{1-\exp \left[-\beta\left(Z-Z_{0}\right)\right]\right\}^{2}+E_{m},
$$

where $\beta$ is a fitting parameter, and

$$
D_{m} \equiv U(\infty, 0)-E_{m} .
$$

Thus $D_{m}=E_{a}(m)-E_{m}$ for $\mathrm{H}_{2}{ }^{+}$, and $D_{m}=E_{a}\left(m_{1}\right)$ $+E_{a}\left(m_{2}\right)-E_{m}$ for $\mathrm{H}_{2}$ (we consider the tightly bound states only). The aligned vibrational energy levels are then given by

$$
E_{n_{\|}}=\hbar \omega_{\|}\left(n_{\|}+\frac{1}{2}\right)-\frac{\left(\hbar \omega_{\|}\right)^{2}}{4 D_{m}}\left(n_{\|}+\frac{1}{2}\right)^{2}
$$


TABLE IV. Bound-state energy levels of $\mathrm{H}_{2}{ }^{+}$. Here $B_{12}=B /\left(10^{12} \mathrm{G}\right), E_{a}$ is the ground-state energy of a $\mathrm{H}$ atom, $m$ is the quantum number specifying the electronic excitations of the molecule, $E_{m}$ is the electronic energy of the molecule, $Z_{0}$ is the equilibrium interatomic separation ( $a_{0}$ is the Bohr radius), $D_{m}$ is defined by $D_{m} \equiv E_{a}(m)-E_{m}, \hbar \omega_{\|}$is the aligned vibrational energy quanta, and $\hbar \omega_{\perp 0}$ is the transverse vibrational energy quanta (neglecting the magnetic forces on protons).

\begin{tabular}{lccccccc}
\hline \hline$B_{12}$ & $E_{a}(\mathrm{eV})$ & $m$ & $E_{m}(\mathrm{eV})$ & $Z_{0}\left(\right.$ units of $\left.a_{0}\right)$ & $D_{m}(\mathrm{eV})$ & $\hbar \omega_{\|}(\mathrm{eV})$ & $\hbar \omega_{\perp 0}(\mathrm{eV})$ \\
\hline 0.1 & -76.4 & 0 & -99.9 & 0.62 & 23.5 & 2.0 & 3.1 \\
0.5 & -130.2 & 0 & -182 & 0.35 & 51.8 & 4.9 & 9.8 \\
1 & -161.5 & 0 & -232 & 0.28 & 70.5 & 6.6 & 16 \\
& & 1 & -162 & 0.40 & 44.8 & 4.4 & \\
2 & -198.5 & 0 & -293 & 0.23 & 94.6 & 9.0 & 25 \\
& & 1 & -207 & 0.32 & 61.5 & 5.9 & \\
5 & -257.1 & 0 & -393 & 0.18 & 136 & 13 & 45 \\
& & 1 & -284 & 0.24 & 91.1 & 8.6 & \\
10 & -309.6 & 0 & -486 & 0.15 & 176 & 17 & 70 \\
& & 1 & -356 & 0.19 & 121 & 12 & \\
\hline \hline
\end{tabular}

where the vibrational energy quanta is

$$
\hbar \omega_{\|}=\hbar \beta\left(\frac{2 D_{m}}{\mu}\right)^{1 / 2} .
$$

The values of $\hbar \omega_{\|}$and $D_{m}$ for different bound electronic states and different magnetic-field strength are given in Table II for $\mathrm{H}_{2}$ and in Table IV for $\mathrm{H}_{2}{ }^{+}$. In Fig. 1, the numerical $E(Z, 0)$ curve is compared with the fitted curve [Eq. (4.2)] for the $(0,1)$ state of $\mathrm{H}_{2}$ at $B_{12}=1$. We see that the fitting is indeed very good, especially for the bound region (below the dark line in Fig. 1). For $\mathrm{H}_{2}$, our results for $\hbar \omega_{\|}$are accurate to within about $5 \%$. The Morse potential fits the $E(Z, 0)$ curves of $\mathrm{H}_{2}^{+}$less well, but the resulting $\hbar \omega_{\|}$is still accurate to within about $10 \%$.

As discussed in Sec. II, $\hbar \omega_{\|}$[cf. Eq. (2.8)] is approximately proportional to $(\ln b)^{2} \mu^{-1 / 2}$ times a slowly increasing function of $B$. Our numerical results confirm this approximate scaling relation. A better empirical scaling is $\hbar \omega_{\|}$ $\propto(\ln b)^{5 / 2} \mu^{-1 / 2}$. Thus for the $\left(m_{1}, m_{2}\right)=(0,1)$ state of $\mathrm{H}_{2}$ $(\mu \simeq 918)$, we have

$\hbar \omega_{\|} \simeq 0.13(\ln b)^{5 / 2} \mu^{-1 / 2}($ a.u. $) \simeq 0.12(\ln b)^{5 / 2}(\mathrm{eV}) \quad\left(\mathrm{H}_{2}\right)$.

For the ground state $(m=0)$ of $\mathrm{H}_{2}{ }^{+}$, we have

$$
\begin{aligned}
\hbar \omega_{\|} & \simeq 0.085(\ln b)^{5 / 2} \mu^{-1 / 2} \text { (a.u.) } \\
& \simeq 0.076(\ln b)^{5 / 2}(\mathrm{eV})\left(\mathrm{H}_{2}{ }^{+}\right) .
\end{aligned}
$$

Both Eqs. (4.6) and (4.7) are accurate to within about $10 \%$. These fitting expressions are indeed very satisfactory considering the approximation introduced when we use the Morse potential to fit the numerical $E(Z, 0)$ curves.

There is no previous reliable calculation for $\mathrm{H}_{2}$ molecules. For $\mathrm{H}_{2}{ }^{+}$, our results for the ground-state electronic energy, interatomic spacing, and aligned vibrational energy quanta $\hbar \omega_{\|}$agree with those obtained by Wunner, Herold, and Ruder [15], and those of Le Guillou and Zinn-Justin [17], who used a similar method as ours in the aligned cases. The slight difference in $\hbar \omega_{\|}$between our results and theirs is likely due to the different ways of extracting this quantity: we obtain it by fitting $E(Z, 0)$ to a Morse potential, while they obtained it by evaluating the second derivative of $E(Z, 0)$ around the equilibrium separation. Le Guillou and Zinn-Justin also considered the effects of nonadiabaticity (i.e., mixing of different electron Landau levels). This is negligible for the field strength of interest in this paper $(b \gg 1)$. The variational calculation of Khersonskii [16] gave somewhat smaller (by about $20 \%$ ) values for $\hbar \omega_{\|}$. This is due to the inaccuracy in his atomic binding energy.

\section{Transverse vibrational excitations}

Neglecting the magnetic forces on the protons, the transverse oscillations of the molecule are governed by the potential $U\left(Z_{0}, R_{\perp}\right)=E\left(Z_{0}, R_{\perp}\right)$. Our calculation of this function is less accurate than the aligned case, and yields only an upper limit to the exact potential. For small-amplitude oscillation (see Sec. II), we fit this potential to a harmonic form

$$
\delta U\left(R_{\perp}\right)=U\left(Z_{0}, R_{\perp}\right)-U\left(Z_{0}, 0\right) \simeq \frac{1}{2} \mu \omega_{\perp 0}^{2} R_{\perp}^{2} .
$$

The transverse vibrational motion of the protons is therefore described by a two-dimensional harmonic oscillator. The numerical values for the transverse vibrational energy quanta are tabulated in Table II (for $\mathrm{H}_{2}$ ) and in Table IV (for $\mathrm{H}_{2}{ }^{+}$). Only the results for the ground electronic states are given.

Note that $\hbar \omega_{\perp 0}$ is larger than $\hbar \omega_{\|}$for $B_{12} \gtrsim 1$. Also, $\hbar \omega_{\perp 0}$ and $\hbar \omega_{\|}$can be comparable or even larger than the electronic excitation energy spacings $\Delta E_{m}$. This is in contrast with the zero-field cases, where one has $\Delta E_{m} \gg \hbar \omega_{v i b}$ $\gg \hbar \omega_{\text {rot }}$. Although the actual values of $\hbar \omega_{\perp 0}$ may be somewhat smaller than our results, the qualitative features revealed in our calculations are expected to be valid in general.

The discussion in Sec. II gives $\hbar \omega_{\perp 0}$ $\sim\left(\xi^{-1} b \ln b\right)^{1 / 2} \mu^{-1 / 2}$ [Eq. (2.10)], where $\xi^{-1}$ increases slowly with increasing $B$. Our numerical results confirm this approximate scaling relation and $\hbar \omega_{\perp 0} \propto b^{1 / 2}(\ln b) \mu^{-1 / 2}$ fits better the results in Table II and Table IV. For the $\left(m_{1}, m_{2}\right)=(0,1)$ state of $\mathrm{H}_{2}$, we have 


$$
\begin{aligned}
\hbar \omega_{\perp 0} & \simeq 0.125 b^{1 / 2}(\ln b) \mu^{-1 / 2} \text { (a.u.) } \\
& =0.553\left(\frac{b}{b_{\text {crit }}}\right)^{1 / 2} \ln b(\text { a.u. })=0.11 b^{1 / 2}(\ln b)(\mathrm{eV})\left(\mathrm{H}_{2}\right) .
\end{aligned}
$$

For the $m=0$ state of $\mathrm{H}_{2}{ }^{+}$, we have

$$
\begin{aligned}
\hbar \omega_{\perp 0} & \simeq 0.14 b^{1 / 2}(\ln b) \mu^{-1 / 2}(\text { a.u. })=0.62\left(\frac{b}{b_{\text {crit }}}\right)^{1 / 2} \ln b(\text { a.u. }) \\
& =0.13 b^{1 / 2}(\ln b)(\mathrm{eV})\left(\mathrm{H}_{2}{ }^{+}\right) .
\end{aligned}
$$

Our results for $\hbar \omega_{\perp 0}$ of $\mathrm{H}_{2}{ }^{+}$also agree closely with those of Le Guillou and Zinn-Justin [17] obtained using their "static approximation," which is similar to ansatz A adopted in our paper (Sec. III B 1). Their improved calculations indicate that the real value of $\hbar \omega_{\perp 0}$ can be lower by tens of percent (from about $10 \%$ for $B_{12}=0.1$ to about $40 \%$ for $B_{12}=5$ ). We expect our results for $\mathrm{H}_{2}$ to have similar accuracy. However, as noted in Sec. II C, the present results apply only to the small-amplitude $\left(R_{\perp} \gtrless \hat{\rho}\right)$ vibrations and relatively weak field $\left(B \gtrsim B_{\text {crit }}\right)$. For sufficiently large field strength, the magnetic forces on the protons become important and can change the transverse vibration energy significantly, as we discuss below.

\section{EFFECTS OF FINITE PROTON MASS ON THE ELECTRONIC ENERGY AND MOLECULAR DISSOCIATION ENERGY}

Our calculations and results in the previous sections are based on Born-Oppenheimer approximation where the proton positions are fixed when we consider the electronic energy of the molecule. For finite proton mass, one can rescale the electronic energy by replacing the electron mass with an appropriate reduced mass. This only introduces a small correction (of order $m_{e} / m_{p}$ ), and is neglected in our paper. However, as noted in Sec. I, the separation of the proton and electron motion in a strong magnetic field is much more complicated, especially in the regime of $B \gtrsim B_{\text {crit }}$ when the cyclotron energy of a proton,

$\hbar \omega_{p}=\hbar\left(e B / m_{p} c\right)=\left(b / b_{c r i t}\right) \ln b_{c r i t}($ a.u. $)=6.3 B_{12}(\mathrm{eV})$,

is comparable with or larger than the spacing of the electronic energy levels. The "standard procedure" for separating the proton and electron motion leads to some ambiguities regarding the binding energy of $\mathrm{H}_{2}$ in the strong field regime; these are discussed in Sec. V A. An alternative scheme, which is more suitable for $B \gtrsim B_{\text {crit }}$, is described in Sec. V B. An approximate expression for the "corrected" dissociation energy of $\mathrm{H}_{2}$ in the ground state is given by Eq. (5.7).

\section{A. Unbound states from the standard scheme when $B \gtrsim B_{\text {crit }}$}

In Secs. II and III we have followed the "standard procedure" for molecules, where one first considers the two protons as infinitely massive fixed at equilibrium separation $Z_{0}$ along the same field line, with their motion included only as an "afterthought." This is strictly valid only for $B \ll B_{\text {crit }}$, where the zero-point vibration amplitudes and the magnetic force on the protons are small. The two electrons, both in the lowest Landau level, are in cylindrical orbitals $m_{1}$ and $m_{2}$ centered on the proton field line with radii given by Eq. (1.2). The Pauli principle requires $m_{1} \neq m_{2}$ so that the ground state has $m_{1}+m_{2}=1$. As the $p$ - $p$ separation $Z$ is allowed to increase, the system tends to two free $\mathrm{H}$ atoms, one in orbital state $m_{1}$ and another $m_{2}$. The standard procedure for treating the two-body problem of a hydrogen atom [20,21] deals with states where the transverse pseudomomentum of each atom is zero, in which case the protons must have Landau excitations $m_{1}$ and $m_{2}$, respectively. The simplest state for the molecule with electronic orbitals $m_{1}$ and $m_{2}$ is then the state where the protons have these Landau excitations at all separations (even though the transverse pseudomomentum is conserved only for the total molecule, not individual atoms). This choice adds a Landau excitation energy $\left(m_{1}+m_{2}\right) \hbar \omega_{p}$, i.e., even the electronic ground state would have an additional positive energy $\hbar \omega_{p}$ and would be unbound (relative to two ground-state atoms) for $B \gg B_{\text {crit }}$. This molecular state has the simplest wave function but not necessarily the lowest energy, since there are states where pseudomomentum is not zero (corresponding to finite separation of the guiding centers of the electron and proton; see Ref. [20]). States without the additional energy $\hbar \omega_{p}$ are discussed in Sec. V B.

For infinite proton mass, the dissociation energy of $\mathrm{H}_{2}$ is given by $D^{(\infty)}=2 E_{a}(0)-E_{m}$. Our numerical results for the $\left(m_{1}, m_{2}\right)=(0,1)$ ground state can be written in the following form:

$$
\begin{array}{r}
D^{(\infty)} \simeq 0.106\left[1+\tau \ln \left(\frac{b}{b_{\text {crit }}}\right)\right](\ln b)^{2} \\
(\text { a.u. }), \\
\tau \simeq 0.1(\ln b)^{0.2},
\end{array}
$$

where $\tau$ varies slowly with $b \quad\left(\tau \simeq 0.14\right.$ for $b \sim 10^{3}$ and $\tau \simeq 0.17$ for $\left.b \sim 10^{5}\right)$. For field strength $b \simeq b_{\text {crit }}$ the square bracket in Eq. (5.2) can be replaced by unity. As shown in Sec. II B and Sec. IV B, the aligned proton vibrations have an energy spacing of order $\hbar \omega_{\|} \sim \mu^{-1 / 2} D^{(\infty)}$ and a small vibration amplitude of order $\delta Z \sim \mu^{-1 / 4} Z_{0}$, where $Z_{0}$ is the equilibrium separation between the protons [Eq. (2.5)]. The inequality $\hbar \omega_{\|} \ll D^{(\infty)}$ does not depend appreciably on the magnetic-field strength, so for the ground molecular state we should be able to use $\hbar \omega_{\|}$in Eq. (4.6) for the aligned vibrations even when $B \gg B_{\text {crit }}$; furthermore, since $\delta Z \ll Z_{0}$, we do not need to consider the Pauli principle explicitly for the transverse wave functions of the protons. For treating this transverse motion, however, the magnetic force becomes important when $b \gtrsim b_{\text {crit }}$, as can be seen from the ratio of the expressions in Eqs. (5.1) and (4.9), $\hbar \omega_{p} / \hbar \omega_{\perp 0} \simeq 1.81\left(b / b_{c r i t}\right)^{1 / 2}\left(\ln b_{c r i t} / \ln b\right)$. We can give at least a plausibility argument for the inclusion into the "standard scheme" of the magnetic effects on the transverse motion from the following consideration: A free proton in the magnetic field $B$ has a zero-point energy $\hbar \omega_{p} / 2$. This can be thought of as the ground-state energy in a "magnetic restoring potential" $(1 / 2) m_{p} \omega_{p}^{2}\left(R_{\perp} / 2\right)^{2}$, which gives a groundstate wave function of size $R_{\perp} \sim \hat{\rho}$ (independent of mass) as in the Landau wave function [Eq. (2.2)]. Thus the total restoring potential for the protons in $\mathrm{H}_{2}$ is given by the sum of the "electronic potential" $\delta U\left(R_{\perp}\right)$, which we have calculated in Sec. III, and twice (for two protons) of the magnetic 
restoring potential. For $R_{\perp} \gtrsim \hat{\rho}$, we have seen that $\delta U\left(R_{\perp}\right)$ can be approximated by the quadratic form in Eq. (4.8), so that the total potential is $\mu\left(\omega_{\perp 0}^{2}+\omega_{p}^{2}\right) R_{\perp}^{2} / 2$. The size of the ground-state wave function is then $\lesssim \hat{\rho}$, the approximation is justified, and the excitation energy quanta is $\hbar\left(\omega_{\perp 0}^{2}+\omega_{p}^{2}\right)^{1 / 2}$. Since the energy of $\hbar \omega_{p} / 2$ also exists in an isolated $\mathrm{H}$ atom, the zero-point energy for the transverse oscillation of the molecule can be written as

$$
\hbar \omega_{\perp}=\hbar\left(\omega_{\perp 0}^{2}+\omega_{p}^{2}\right)^{1 / 2}-\hbar \omega_{p}
$$

For $b \ll b_{\text {crit }}$ we have $\hbar \omega_{\perp} \simeq \hbar \omega_{\perp 0}$ as expected, and Eq. (5.2) shows that $\hbar \omega_{\perp} / D^{(\infty)} \sim 5\left(b / b_{c r i t}\right)^{1 / 2} / \ln b \ll 1$. For $b \gg b_{\text {crit }}$, on the other hand, $\hbar \omega_{\perp} \simeq \hbar \omega_{\perp 0}^{2} /\left(2 \omega_{p}\right) \simeq 0.016 l^{2}$ so that $\hbar \omega_{\perp} / D^{(\infty)} \sim 0.15$. Thus the transverse zero-point energy $\hbar \omega_{\perp}$ remains less than the dissociation energy for the state given by the standard scheme, but the Landau energy $\hbar \omega_{p}$ of the excited proton has to be added to the molecular energy also. The "corrected" dissociation energy in this scheme is then given by

$$
\begin{gathered}
D^{(s t d)}=D^{(\infty)}-\frac{1}{2} \hbar \omega_{\|}-\Delta \varepsilon^{(s t d)}, \\
\Delta \varepsilon^{(s t d)}=\hbar\left(\omega_{\perp 0}^{2}+\omega_{p}^{2}\right)^{1 / 2} .
\end{gathered}
$$

Clearly, $D^{(s t d)}$ becomes negative (i.e., the state is unbound) as $b$ increases beyond $b_{\text {crit }}$. We shall see in Sec. V B that an alternative scheme gives molecular bound states with lower energy for $b \gtrsim b_{\text {crit }}$.

\section{B. The alternative scheme}

The alternative scheme we propose for the $\mathrm{H}_{2}$ molecule ground state is a generalization of the scheme for the $\mathrm{H}$ atom described in Sec. IV of Ref. [20]. In this scheme the transverse pseudomomentum is not chosen as a good quantum number, and our approximate wave function will not be an exact eigenstate of the Hamiltonian. However, it does provide a suitable trial wave function and enables us to obtain a rigorous lower limit to the dissociation energy $D$. In the absence of Coulomb interaction between the four particles, there are eight quantum numbers specifying the transverse degrees of freedom of the system: the Landau excitation number $n$ and the orbital number $m$ for each of the four particles. For the $\mathrm{H}_{2}$ ground state we then choose $n=0$ for both electrons and both protons (so there is no $\hbar \omega_{p}$ contribution to the electronic energy of the molecule), and $\left(m_{1}, m_{2}\right)=(0,1)$ for the electrons. We can choose $m=0$ for both protons since, as mentioned, the proton $z$ wave function can be antisymmetrized to satisfy the Pauli principle with little energy contribution. As a trial wave function we assume that the charge distribution of protons consists of two sheets separated in the $z$ axis by distance $Z$, with surface density given by $\left|W_{0}\left(\mathbf{r}_{\perp}\right)\right|^{2}$. Obviously, when the Coulomb potentials between the particles are restored, $n$ and $m$ for the individual particle cease to be good quantum numbers, ${ }^{4}$ but the trial wave function thus constructed will give an upper bound to the true ground-state energy of the molecule according to the variational principle. This "trial energy" can be calculated using the Hartree-Fock method described in Sec. II A, subjected to two modifications: (i) the averaged electronproton interaction potential $\tilde{V}_{m}(z)$ in Eq. (3.5) is replaced by

$$
\begin{aligned}
\tilde{V}_{m}(z) \rightarrow & \int d^{2} \mathbf{r}_{\perp e}\left|W_{m}\left(\mathbf{r}_{\perp e}\right)\right|^{2} \int d^{3} \mathbf{r}_{p}\left|W_{0}\left(\mathbf{r}_{\perp p}\right)\right|^{2} \\
& \times\left[\delta\left(z_{p}-\frac{Z}{2}\right)+\delta\left(z_{p}+\frac{Z}{2}\right)\right] \frac{1}{\left|\mathbf{r}_{e}-\mathbf{r}_{p}\right|} \\
= & D_{0 m}\left(z-\frac{Z}{2}\right)+D_{0 m}\left(z+\frac{Z}{2}\right)
\end{aligned}
$$

where $D_{0 m}(z)$ is defined in Eq. (3.17); (ii) the proton-proton interaction term $e^{2} / Z$ in Eq. (3.19) is replaced by $D_{0 m}(Z)$ (although this modification has a negligible effect on the energy except when $Z \rightarrow 0$ ).

The molecular energy $E_{m}^{(a l t)}$ obtained by this alternative scheme is larger than the result $E_{m}$ obtained using the scheme of Sec. III A (where the protons are treated as infinitely massive), by some amount $\Delta \varepsilon^{(a l t)}$. The weakening of the electron-proton interaction is due to the spread of the proton wave function by an amount of order $\hat{\rho}$. However, since $Z \gg \hat{\rho}$ the change involves only the logarithm of the Coulomb energy. This can be characterized by changing $b$ in Eq. (4.1) to $b /(2 C)$, where $C$ is of order unity, i.e., $E_{m}^{(a l t)} \simeq-0.091[\ln (b / 2 C)]^{2.7}$. To leading order in $\ln (2 C)$, we then have

$$
\begin{gathered}
D^{(a l t)}=D^{(\infty)}-\frac{1}{2} \hbar \omega_{\|}-\Delta \varepsilon^{(a l t)} ; \\
\Delta \varepsilon^{(a l t)} \simeq 0.24 \ln (2 C)(\ln b)^{1.7} \text { (a.u.), }
\end{gathered}
$$

as an alternative to Eq. (5.4).

We have performed numerical calculations and found that the "trial" ground-state energy thus obtained agrees with the result using the scheme of Sec. III A to within $15 \%$. For $B_{12}=50,100,500,10^{3}$, we found $\Delta \varepsilon^{(a l t)} \simeq 162,191,258$, $294 \mathrm{eV}$, corresponding to $C \simeq 0.8$ for $B_{12}=50$ and $C \simeq 0.9$ for $B_{12}=10^{3}$. The numerical values for $\Delta \varepsilon^{(a l t)}$ can be fitted by $\Delta \varepsilon^{(a l t)} \simeq 0.06(\ln b)^{2}$. This has the same scaling with $b$ as $\hbar \omega_{\perp}$ defined in Eq. (5.3). With this value of $\Delta \varepsilon^{(a l t)}$, the dissociation energy given by Eq. (5.6) is larger than that from Eq. (5.4), and therefore represents the true molecular ground state for all $b \gtrsim b_{\text {crit }}$. Our numerical calculation of $\Delta \varepsilon^{(a l t)}$ used a particularly simple trial wave function and a better wave function with the variational method would presumably lower $\Delta \varepsilon^{(a l t)}$ somewhat. This would lower the nu-

\footnotetext{
${ }^{4}$ The only good quantum number for the transverse degrees of freedom is the total orbital angular momentum along the $z$ axis, $L_{z}=\sum_{i} \operatorname{sgn}\left(e_{i}\right)\left(m_{i}-n_{i}\right)$, where $\operatorname{sgn}\left(e_{i}\right)=1$ for proton and $\operatorname{sgn}\left(e_{i}\right)=-1$ for electron [20]. For $b \gg b_{c r i t}$, the Landau excitation numbers $n$ for both electrons and protons are "adiabatically" conserved and can be set to 0 for the ground state. In this case $L_{z}=m_{1 p}+m_{2 p}-m_{1 e}-m_{2 e}$. Thus the true ground state of the molecule for $B \gg B_{\text {crit }}$ involves a mixing of many different $\left(m_{1 e}, m_{2 e}, m_{1 p}, m_{2 p}\right)$ states with the same $L_{z}$.
} 
TABLE V. The dissociation energy of a $\mathrm{H}_{2}$ molecule in the ground state in a superstrong magnetic field. $D^{(\infty)}$ is the dissociation energy assuming infinite proton mass, while $D$ includes the (approximate) correction of the molecular zero-point energy [Eq. (5.7)]. $B_{12}=B /\left(10^{12} \mathrm{G}\right), \hbar \omega_{\|} / 2$ is the zero-point energy for the aligned vibration, and $\hbar \omega_{\perp}$ is the zero-point energy for the transverse vibration [Eq. (5.3)]. Note that for $B_{12}<0.2$, the ground state is actually the "weakly bound" state (see Secs. II A and IV A), and the zero-point energy has been neglected. All energies are expressed in $\mathrm{eV}$.

\begin{tabular}{lccccccc}
\hline \hline$B_{12}$ & 0.1 & 0.5 & 1 & 5 & 10 & 100 & 500 \\
\hline$D^{(\infty)}$ & 14 & 31 & 46 & 109 & 150 & 378 & 615 \\
$D$ & $\simeq 14$ & 21 & 32 & 79 & 110 & 311 & 523 \\
$\hbar \omega_{\|} / 2$ & & 3.6 & 4.9 & 9.5 & 12 & 22 & 31 \\
$\hbar \omega \perp$ & & 6.1 & 9.1 & 21 & 28 & 45 & 61 \\
\hline \hline
\end{tabular}

merical value of $b$ above which the true ground state is the state with no Landau excitations for either proton, obtained by the present "alternative scheme."

The fact that $\Delta \varepsilon^{(a l t)}$ scales similarly with $b$ as $\hbar \omega_{\perp}$ suggests that for practical purposes, the "corrected" dissociation energy of $\mathrm{H}_{2}$ in the ground state can be approximated by

$$
D \simeq D^{(\infty)}-\left(\frac{1}{2} \hbar \omega_{\|}+\hbar \omega_{\perp}\right)
$$

for all field regimes $(b \gg 1)$, where $\hbar \omega_{\perp}$ is given by Eq. (5.3). The numerical results for a wide range of field strength are summarized in Table V.

\section{CONCLUSIONS}

In this paper, we have studied and characterized the energy excitation levels of a $\mathrm{H}_{2}$ molecule in a superstrong magnetic field $\left(B \gtrsim 10^{12} \mathrm{G}\right.$ ) which exists on the surfaces of many neutron stars. The main theoretical uncertainty of our calculations lies in the nontrivial separation of the motion of the protons and that of the electrons. Nevertheless, we find that in such a strong magnetic field, the $\mathrm{H}_{2}$ molecule exhibits completely different energy excitation levels as compared to its well-known zero-field counterpart. The fact that the excitation energies associated with the oscillations of the protons are comparable to the electronic excitations indicates that the statistical weight of a $\mathrm{H}_{2}$ molecule is not much larger than that of a $\mathrm{H}$ atom. This greatly simplifies the calculations of the chemical equilibria of various forms of $\mathrm{H}$ in a neutron star atmosphere [18].

Larger hydrogen molecules and chains can also form in a superstrong magnetic field. Their ground-state binding energies have been calculated in paper I. It is expected that these larger molecules possess qualitatively similar energy excitation levels as those of $\mathrm{H}_{2}$ considered in this paper, with one exception: For a long chain molecule $\mathrm{H}_{n}$ with $1 \ll n$ $\ll\left[b /(\ln b)^{2}\right]^{1 / 5}$, the spacing $Z_{0}$ along a field line between adjacent protons decreases with increasing $n$ approximately as $n^{-2}$. The fractional zero-point vibration amplitude $\Delta Z / Z_{0}$ is of order $\left(m_{e} / m_{p}\right)^{1 / 4} n^{1 / 2}$. The aligned vibrations thus become more pronounced as $n$ increases (and can lead to "internal pycnonuclear reactions" which will be discussed in [18]).
There is no question that the exotic molecules considered in this paper exist on the surfaces of some neutron stars with $B_{12} \gtrsim 10^{12} \mathrm{G}$ and temperature $T \sim 10^{5}-10^{6} \mathrm{~K}$ [18]. For very low surface temperature $\left(T \gtrsim 10^{5} \mathrm{~K}\right)$, the atmosphere is likely to condensate into a metallic state, since the hydrogen metal has the largest binding energy. However, for the astrophysically more interesting temperature range $\left(T \gtrsim 10^{5} \mathrm{~K}\right)$, the outer layer of a neutron star will exist predominantly in the form of nondegenerate gas of individual atoms and small molecules: e.g., when $T \sim 3 \times 10^{5} \mathrm{~K}$, the photosphere of a neutron star is dominated by atoms if $B_{12}=1$, while it is dominated by $\mathrm{H}_{2}$ if $B_{12}=10$. The existence of $\mathrm{H}_{2}$ in the atmosphere will give rise to appreciable radiative opacity. For example, since the proton separation in $\mathrm{H}_{2}$ is different from that in $\mathrm{H}_{2}{ }^{+}$(see Table II and Table IV), the photoionization cross section from the ground state of $\mathrm{H}_{2}$ is expected to be small according to the Franck-Condon principle. However, photoionization from an excited vibrational state or electronic state, for which the proton separation is close to that in the $\mathrm{H}_{2}{ }^{+}$ground state, can provide significant continuum opacity. These issues may warrant further study, especially in light of the increasing possibility of the spectroscopic studies of isolated neutron stars by future $\mathrm{x}$-ray and EUV satellites.

\section{ACKNOWLEDGMENTS}

This work has been supported in part by NSF Grants Nos. AST 91-19475 and AST 93-15375, and NASA Grant No. NAGW-666 to Cornell University and by NASA Grant No. NAGW-2394 to Caltech. D.L. also acknowledges the financial support of the Richard C. Tolman Research Fund in theoretical astrophysics at Caltech.

\section{APPENDIX A: COULOMB INTEGRALS FOR THE LANDAU WAVE FUNCTION}

In this appendix, we derive Eqs. (3.24) and (3.38). First consider the function

$$
V_{m m}\left(z, r_{0}\right)=\left\langle m\left|\frac{1}{\left|\mathbf{r}-\mathbf{r}_{0}\right|}\right| m\right\rangle
$$

with $\mathbf{r}_{\mathbf{0}}=r_{0} \hat{\mathbf{x}}$. Since

$$
\frac{1}{r}=\frac{1}{2 \pi^{2}} \int \frac{d^{3} q}{q^{2}} e^{i \mathbf{q} \cdot \mathbf{r}}
$$

we have

$V_{m m}\left(z, r_{0}\right)=\frac{1}{2 \pi^{2}} \int \frac{d^{3} q}{q^{2}} e^{-i \mathbf{q} \cdot \mathbf{r}_{0}} e^{i q_{z} z}\left\langle m\left|e^{i \mathbf{q}_{\perp} \cdot \mathbf{r}_{\perp}}\right| m\right\rangle$.

Using the general result for the matrix element [32]

$$
\begin{aligned}
\left\langle m^{\prime}\left|e^{i \mathbf{q}_{\perp} \cdot \mathbf{r}_{\perp}}\right| m\right\rangle= & (-1)^{m} i^{m+m^{\prime}}\left(\frac{m !}{m^{\prime} !}\right)^{1 / 2} e^{-q_{\perp}^{2} / 2} L_{m}^{m^{\prime}-m}\left(\frac{q_{\perp}^{2}}{2}\right) \\
& \times\left(\frac{q_{\perp}}{\sqrt{2}}\right)^{m^{\prime}-m} e^{i\left(m^{\prime}-m\right) \theta_{q}} \quad\left(m^{\prime} \geqslant m\right), \quad(\mathrm{A} 4)
\end{aligned}
$$


where $\theta_{q}$ specifies the angle of $\mathbf{q}_{\perp}$ in the $q_{x}-q_{y}$ plane, and $L_{n}^{m}$ is the Laguerre polynomial of order $n[30]$, we have

$$
\left\langle m\left|e^{i \mathbf{q}_{\perp} \cdot \mathbf{r}_{\perp}}\right| m\right\rangle=e^{-q_{\perp}^{2} / 2} L_{m}\left(\frac{q_{\perp}^{2}}{2}\right) .
$$

Substitute Eq. (A5) into (A3), and integrate out $d q_{z}$ and $d \theta_{q}$ using

$$
\int \frac{d q_{z}}{q_{z}^{2}+q_{\perp}^{2}} e^{i q_{z} z}=\frac{\pi}{q_{\perp}} e^{-q_{\perp}|z|}
$$

and

$$
\int d \theta_{q} e^{-i q_{\perp} r_{0} \cos \theta_{q}}=2 \pi J_{0}\left(q_{\perp} r_{0}\right),
$$

we obtain

$$
V_{m m}\left(z, r_{0}\right)=\int_{0}^{\infty} d q_{\perp} e^{-q_{\perp}^{2} / 2-q_{\perp}|z|} J_{0}\left(q_{\perp} r_{0}\right) L_{m}\left(\frac{1}{2} q_{\perp}^{2}\right),
$$

i.e., Eq. (3.24). Note that using Eq. (A4), a more general expression can be obtained for the matrix element

$$
\begin{aligned}
V_{m^{\prime} m}\left(z, r_{0}\right) \equiv & \left\langle m^{\prime}\left|\frac{1}{\left|\mathbf{r}-\mathbf{r}_{0}\right|}\right| m\right\rangle \\
= & \left(\frac{m !}{m^{\prime} !}\right)^{1 / 2} \int_{0}^{\infty} d q\left(\frac{q}{\sqrt{2}}\right)^{m^{\prime}-m} \\
& \times e^{-q^{2} / 2-q|z|} J_{m^{\prime}-m}\left(q r_{0}\right) L_{m}^{m^{\prime}-m}\left(\frac{1}{2} q^{2}\right) \\
& \left(m^{\prime} \geqslant m\right) .
\end{aligned}
$$

Next consider $\tilde{D}_{m_{1} m_{2}}$ defined in Eq. (3.36). Changing variable $\left(\mathbf{r}_{2 \perp}-\mathbf{R}_{\perp}\right) \rightarrow \mathbf{r}_{2 \perp}$, we have

$$
\begin{aligned}
\tilde{D}_{m_{1} m_{2}}\left(z_{1}-z_{2}, R_{\perp}\right)= & \int d^{2} \mathbf{r}_{1 \perp} d^{2} \mathbf{r}_{\mathbf{2}}\left|W_{m_{1}}\left(\mathbf{r}_{1 \perp}\right)\right|^{2} \\
& \times\left|W_{m_{2}}\left(\mathbf{r}_{2 \perp}\right)\right|^{2} \frac{1}{\left|\mathbf{r}_{1}-\mathbf{r}_{2}-\mathbf{R}_{\perp}\right|} .
\end{aligned}
$$

Using Eq. (A2), we have

$$
\begin{aligned}
\tilde{D}_{m_{1} m_{2}}\left(z, R_{\perp}\right)= & \frac{1}{2 \pi^{2}} \int \frac{d^{3} q}{q^{2}} e^{-i \mathbf{q} \cdot \mathbf{R}_{\perp}} e^{i q_{z} z}\left\langle m_{1}\left|e^{i \mathbf{q}_{\perp} \cdot \mathbf{r}_{\perp}}\right| m_{1}\right\rangle \\
& \times\left\langle m_{2}\left|e^{i \mathbf{q}_{\perp} \cdot \mathbf{r}_{\perp}}\right| m_{2}\right\rangle .
\end{aligned}
$$

Again, using Eq. (A4), and integrating out $d q_{z}$ and $d \theta_{q}$ with Eqs. (A6) and (A7), we obtain

$$
\tilde{D}_{m_{1} m_{2}}\left(z, R_{\perp}\right)=\int_{0}^{\infty} d q e^{-q^{2}-q|z|} J_{0}\left(q R_{\perp}\right) L_{m_{1}}\left(\frac{1}{2} q^{2}\right)
$$

$$
L_{m_{2}}\left(\frac{1}{2} q^{2}\right)
$$

Now defining the coefficient $d_{s}\left(m_{1}, m_{2}\right)$ via (see paper I)

$$
L_{m_{1}}\left(\frac{x}{2}\right) L_{m_{2}}\left(\frac{x}{2}\right)=\sum_{s=0}^{m_{1}+m_{2}} d_{s}\left(m_{1}, m_{2}\right) L_{s}(x),
$$

Eq. (A12) then becomes

$$
\begin{aligned}
\tilde{D}_{m_{1} m_{2}}\left(z, R_{\perp}\right)= & \sum_{s=0}^{m_{1}+m_{2}} d_{s}\left(m_{1}, m_{2}\right) \\
& \times \int_{0}^{\infty} d q e^{-q^{2}-q|z|} J_{0}\left(q R_{\perp}\right) L_{s}\left(q^{2}\right) \\
= & \sum_{s=0}^{m_{1}+m_{2}} d_{s}\left(m_{1}, m_{2}\right) \\
& \times \int_{0}^{\infty} \frac{d q}{\sqrt{2}} e^{-q^{2} / 2-q|z| / \sqrt{2}} J_{0}\left(\frac{q R_{\perp}}{\sqrt{2}}\right) L_{s}\left(\frac{q^{2}}{2}\right),
\end{aligned}
$$

which reduces to Eq. (3.38) after using Eq. (A8).

\section{APPENDIX B: MORE ACCURATE CALCULATION OF $\mathrm{H}_{2}^{+}$}

An "exact" treatment of $\mathrm{H}_{2}{ }^{+}$for general orientation of the molecular axis proceeds as follows. Consider the coordinate system of ansatz A in Sec. III B 1. When $b \gg 1$, the most general electron wave function for the $\nu=0$ state can be written as

$$
\Phi_{m 0}(\mathbf{r})=\sum_{m} W_{m}\left(\mathbf{r}_{\perp}\right) f_{m 0}(z)
$$

Substituting this into the Schrödinger equation and averaging over the transverse direction, we obtain a set of differential equations for $f_{m 0}(z)$ :

$$
\begin{aligned}
- & \frac{\hbar^{2}}{2 m_{e} \hat{\rho}^{2}} \frac{d^{2}}{d z^{2}} f_{m 0}(z)-\frac{e^{2}}{\hat{\rho}} \sum_{m^{\prime}} \tilde{V}_{m m^{\prime}}\left(z, R_{\perp / 2}\right) f_{m^{\prime} 0}(z) \\
& =\varepsilon_{m 0} f_{m 0}(z), \quad m=0,1, \ldots,
\end{aligned}
$$

where $V_{m m^{\prime}}$ is defined similar to Eq. (3.23):

$$
\tilde{V}_{m m^{\prime}}\left(z, R_{\perp} / 2\right)=V_{m m^{\prime}}\left(\left|z-\frac{Z}{2}\right|, \frac{R_{\perp}}{2}\right)+V_{m m^{\prime}}\left(\left|z+\frac{Z}{2}\right|, \frac{R_{\perp}}{2}\right),
$$

and the function $V_{m m^{\prime}}$ can be evaluated using Eq. (A9). Equation (B2) is subject to the boundary conditions 
$d f_{m 0} / d z=0$ at $z=0$ and $f_{m 0} \rightarrow 0$ as $z \rightarrow \infty$. The normalization condition requires

$$
\sum_{m} \int_{-\infty}^{\infty} d z\left|f_{m 0}(z)\right|^{2}=1
$$

The set of equations (B2) can be solved numerically using an iterative scheme similar to that used for solving the Hartree-Fock equation (paper I). Successively accurate re- sults can be obtained by using an increasing number of terms in the sum in Eq. (B1). The lowest energy state corresponds to the solution satisfying

$$
\int_{-\infty}^{\infty} d z\left|f_{00}(z)\right|^{2}>\int_{-\infty}^{\infty} d z\left|f_{10}(z)\right|^{2}>\int_{-\infty}^{\infty} d z\left|f_{20}(z)\right|^{2}>\cdots .
$$

Generalization of this method to a $\mathrm{H}_{2}$ molecule is much more complicated.
[1] L. I. Schiff and H. Snyder, Phys. Rev. 55, 59 (1939).

[2] R. J. Elliot and R. Loudon, J. Phys. Chem. Solids 15, 196 (1960); H. Hasegawa and R. E. Howard, ibid. 21, 179 (1961).

[3] M. Ruderman, in Physics of Dense Matter, International Astronomical Union Symposium No. 53, edited by C. J. Hansen (North-Holland, Dordrecht; 1974).

[4] H. Ruder, G. Wunner, H. Herold, and F. Geyer, Atoms in Strong Magnetic Fields (Springer-Verlag, Berlin, 1994).

[5] H. Ögelman, in Physics of Isolated Pulsars, edited by K. van Riper, C. Ho, and R. Epstein (Cambridge University Press, Cambridge, 1992).

[6] D. Lai, E. E. Salpeter, and S. L. Shapiro, Phys. Rev. A 45, 4832 (1992) (paper I).

[7] A. R. P. Rau, R. O. Mueller, and L. Spruch, Phys. Rev. A 11, 1865 (1975); E. G. Flowers, J. F. Lee, M. A. Ruderman, P. G. Sutherland, W. Hillebrandt, and E. Müller, Astrophys. J. 215, 291 (1977); E. Müller, Astron. Astrophys. 130, 415 (1984).

[8] For a review of the Thomas-Fermi model in strong magnetic field, see I. Fushiki, E. H. Gudmundsson, C. J. Pethick, and J. Yngvason, Ann. Phys. (N.Y.) 216, 29 (1992). See also A. M. Abrahams and S. L. Shapiro, Astrophys. J. 382, 233 (1991); E. H. Lieb, J. P. Solovej, and J. Yngvason, Phys. Rev. Lett. 69, 749 (1992); Commun. Pure Appl. Math. 47, 513 (1994).

[9] P. B. Jones, Mon. Not. R. Astron. Soc. 216, 503 (1985).

[10] J. Virtamo, J. Phys. B 9, 751 (1976); P. Pröschel, W. Rösner, G. Wunner, H. Ruder, and H. Herold, ibid. 15, 1959 (1982).

[11] D. Neuhauser, S. E. Koonin, and K. Langanke, Phys. Rev. A 36, 4163 (1987).

[12] M. C. Miller and D. Neuhauser, Mon. Not. R. Astron. Soc. 253, 107 (1991).

[13] W. Rösner, G. Wunner, H. Herold, and H. Ruder, J. Phys. B 17, 29 (1984); S. P. Goldman and Z. Chen, Phys. Rev. Lett. 67, 1403 (1991).

[14] D. M. Larsen, Phys. Rev. A 25, 1295 (1982).

[15] G. Wunner, H. Herold, and H. Ruder, Phys. Lett. 88A, 344 (1982).

[16] V. K. Khersonskii, Astrophys. Space Sci. 98, 255 (1984); 117, 47 (1985).
[17] J. C. Le Guillou and J. Zinn-Justin, Ann. Phys. (N.Y.) 154, 440 (1984).

[18] D. Lai and E. E. Salpeter, Astrophys. J. (to be published).

[19] D. Lai, E. E. Salpeter, and S. L. Shapiro, in Physics of Isolated Pulsars (Ref. [5]).

[20] The two-body problem in a strong magnetic field has been discussed and reviewed in D. Lai and E. E. Salpeter, Phys. Rev. A. 52, 2611 (1995).

[21] J. E. Avron, I. B. Herbst, and B. Simon, Ann. Phys. (N.Y.) 114, 431 (1978); H. Herold, H. Ruder, and G. Wunner, J. Phys. B 14, 751 (1981); A. E. Shabad and V. V. Usov, Astron. Space Sci. 128, 377 (1986).

[22] Born-Oppenheimer approximation in a strong magnetic field is discussed in P. Schmelcher, L. S. Cederbaum, and H.-D. Meyer, Phys. Rev. A 38, 6066 (1988). Also see B. R. Johnson, J. O. Hirschfelder, and K.-H. Yang, Rev. Mod. Phys. 55, 109 (1983) for a review.

[23] C. Angelie and C. Deutch, Phys. Lett. 67A, 353 (1978).

[24] L. K. Haines and D. H. Roberts, Am. J. Phys. 37, 1145 (1969).

[25] A. V. Korolev and M. A. Liberman, Phys. Rev. Lett. 72, 270 (1994); Phys. Rev. B 47, 14318 (1993); Physica A 193, 347 (1993); Phys. Rev. A 45, 1762 (1992).

[26] D. Lai, Phys. Rev. Lett. 74, 4095 (1995).

[27] G. Ortiz, M. D. Jones, and D. M. Ceperley, Phys. Rev. A (to be published).

[28] L. D. Landau and E. M. Lifshitz, Quantum Mechanics (Pergamon, Oxford, 1977).

[29] J. C. Slater, Quantum Theory of Molecules and Solids, Vol. 1 (McGraw-Hill, New York, 1963).

[30] M. Abramowitz and I. Stegun, Handbook of Mathematical Functions (Dover, New York, 1972).

[31] W. H. Press, B. P. Flannery, S. A. Teukolsky, and W. T. Vetterling, Numerical Recipes: The Art of Scientific Computing (Cambridge University Press, Cambridge, 1987).

[32] J. Virtamo and P. Jauho, Nuovo Cimento 26B, 537 (1975); A. A. Sokolov and I. M. Ternov, Synchrotron Radiation (Perga mon, Oxford 1968). 